\title{
Estudio espectroscópico y DRX de afloramientos terrestres volcánicos en la isla de Tenerife como posibles análogos de la geología marciana
}

\section{Spectroscopic analysis and XRD of terrestrial volcanic outcrops on the Tenerife Island as possible Martian analogue}

\author{
E.A. Lalla ${ }^{1,2}$, G. López-Reyes ${ }^{2}$, A. Sansano², A. Sanz-Arranz ${ }^{2}$, D. Schmanke ${ }^{3}$, \\ G. Klingelhöfer ${ }^{3}$, J. Medina-García ${ }^{2}$, J. Martínez-Frías ${ }^{4}$, y F. Rull-Pérez ${ }^{2}$ \\ 1 Departamento de Física, Facultad de Ciencias, Universidad de La Laguna, Tenerife, España. Email: lallaea@cab.inta-csic.es \\ 2 Unidad Asociada UVa-CSIC al Centro de Astrobiología, Parque Tecnológico de Boecillo, Valladolid, España \\ 3 Universidad de Johannes Gutenberg, Mainz, Alemania \\ 4 Departamento de Dinámica Terrestre y Observación de la Tierra, Instituto de Geociencias (CSIC, UCM), Madrid, España
}

\section{RESUMEN}

Se ha llevado a cabo una selección de varios afloramientos volcánicos en la Isla de Tenerife (La Caldera de las Cañadas, vulcanismo histórico y la zona del Malpaís de Güimar) como posibles análogos terrestres de Marte, considerando los procesos volcánicos ocurridos durante algunas de las etapas geológicas del planeta rojo. En la selección de las áreas de estudio se han tenido en cuenta la diversidad de procesos de alteración, que incluyen fenómenos como meteorización y alteración hidrotermal. Estos procesos terrestres podrían servir como modelo de la actividad volcánica primitiva en Marte.

Los materiales seleccionados se han analizado mediante espectroscopia micro-Raman, difracción de rayos $\mathrm{X}$ (DRX) y espectroscopia Mössbauer. Los resultados revelan que la mineralogía de los afloramientos está constituida por una matriz de olivinos, piroxenos y feldespatos. Además, se ha detectado una gran variedad de especies minerales correspondientes a procesos de alteración como óxidos, arcillas y carbonatos.

Los resultados obtenidos han demostrado ser un punto de partida para desarrollar investigaciones en estos entornos volcánicos especialmente enfocados al desarrollo de la ciencia de instrumentación para exploración planetaria. La instrumentación utilizada en la investigación ha sido a través de prototipos gemelos empleados en la exploración espacial, como DRX (actualmente en la misión NASA-MSL-Curiosity), espectroscopia Mössbauer (a bordo de la misión NASA-MER) y el futuro instrumento Raman de la misión ESA-ExoMars. Además, la mineralogía detectada coincide con los resultados reportados en Marte.

Los métodos de análisis Raman, tanto de caracterización e identificación, mediante el estudio de patrones espectrales se han aplicado exitosamente, donde el análisis de los perfiles Raman son de extrema utilidad para aclarar el origen de las especies minerales. La espectroscopia Mössbauer y la difracción de rayos X han confirmado los resultados Raman.

Palabras Clave: Vulcanología; Espectroscopia Raman; Espectroscopia Mössbauer; Difracción de Rayos X; Marte; Tenerife.

Recibido el 15 de octubre de 2014 / Aceptado el 4 de mayo de 2015 / Publicado online el 03 de noviembre de 2015

Citation / Cómo citar este artículo: E.A. Lalla, et al. (2015). Estudio espectroscópico y DRX de afloramientos terrestres volcánicos en la isla de Tenerife como posibles análogos de la geología marciana. Estudios Geológicos 71(2): e035. http://dx.doi.org/10.3989/ egeol.41927.354.

Copyright: () 2015 CSIC. This is an open-access article distributed under the terms of the Creative Commons Attribution-Non Commercial (by-nc) Spain 3.0 License. 


\section{ABSTRACT}

Several volcanic outcrops from Tenerife Island (Las Cañadas Caldera, historical volcanism and Güimar-Malpaís outcrop) has been selected as a potential terrestrial analog for Mars, regarding the Martian mineralogy and its volcanic characteristic. Diverse alteration processes, including weathering and hydrothermal alteration have been detected in these volcanics environments, which could be considered as part of a model for the primitive volcanic activity of Mars.

The selected materials have been measured by micro-Raman spectroscopy, XRD and Mössbauer spectroscopy. The results show the primary mineralogy comprises on olivine, pyroxene and feldspar. Also, a wide variety of alterational materials, including oxides, clay minerals and carbonates have been detected.

The results have proven to be a starting point to develop research focused to the development of science instrumentation for planetary exploration in volcanic environments. The instrumentation used was primarily through twin prototypes applied in space exploration such as XRD (on board at the NASA-MSL-Curiosity mission), Mössbauer spectroscopy (on board at the NASA-MER mission) and the future Raman instrument on ESA mission -ExoMars. In addition, the detected mineralogy is consistent with results reported on Mars. The Raman spectral analisys methods, characterization and identification, have been applied, where the analysis of the Raman profiles are extremely useful to clarify the geochemical origin of the mineral species. On the other hand, the results obtained by Raman have been confirmed by Mössbauer spectroscopy and X-ray diffraction.

Keywords: Volcanology; Raman Spectroscopy; Mössbauer Spectroscopy; X Ray Diffraction; Mars; Tenerife.

\section{Introducción}

El éxito de las misiones planetarias depende del desarrollo de la ciencia asociada a los instrumentos a bordo de los orbitadores, "landers" o "rovers". Mejoras relacionadas con la calibración de los instrumentos, el análisis de materiales en condiciones marcianas simuladas y el estudio de las limitaciones y virtudes de las técnicas para estudiar minerales a distintas escalas son algunas de las líneas de investigación que se están llevando a cabo (Rull \& Frías, 2006; Vago, 2006). El estudio preliminar de minerales en afloramientos terrestres considerados "análogos terrestres de Marte" supone un paso más a la hora de explorar las capacidades analíticas de las técnicas implicadas en misiones planetarias. (Lalla et al., 2010). Se define un análogo terrestre como un afloramiento que presenta condiciones geológicas o medioambientales similares a las halladas en otro cuerpo extraterrestre (West et al., 2010). En el caso concreto de Marte es de especial relevancia el estudio de zonas con características geomorfológicas y procesos similares a los desarrollados en Marte, fundamentalmente de carácter volcánico. Entre estos procesos geológicos cabe destacar: (1) Los desarrollados en ambientes áridos y zonas polares con gran actividad de procesos eólicos, periglaciares, evaporíticos o de alteración; (2) los procesos volcánico-glaciares con erupciones efusivas y procesos hidrotermales; y (3) los procesos magmáticos relacionados con la formación de rocas (Osinski et al., 2006; West et al., 2010).
Las islas volcánicas jóvenes, como las islas Canarias, conforman un entorno apropiado para abordar este objetivo ya que se ha propuesto que, en determinados aspectos, podrían servir de análogos para comprender algunos procesos geológicos desarrollados en el pasado de Marte (Carracedo, 1999; Lalla et al., 2011). Uno de los sitios idóneos para llevar a cabo este tipo de estudios comparativos es la isla de Tenerife (Figura 1), en la que se pueden observar afloramientos basálticos correspondientes a las primeras etapas de formación de la isla (Bustillo \& Martínez-Frías, 2003; Rodríguez-Losada et al., 2000). Existe mucha y variada información sobre el origen del archipiélago de las Canarias y sus diferentes hipótesis genéticas (véase, entre otros, Araña \& Ortiz, 1986; Anguita \& Hernán, 1975; Carracedo, 1999, Anguita \& Hernán, 2000). El magmatismo de Canarias en general, y el de Tenerife en particular, se caracteriza fundamentalmente por su naturaleza alcalina y la presencia de materiales como las traquitas y fonolitas y ocasionalmente por la aparición de rocas con tendencias toleíticas (Carracedo, 1975; Thirlwall et al., 2000). Con respecto a los procesos de alteración, aparecen una gran variedad de entornos geológicos que albergan o albergaron procesos de alteración submarina, meteorización e interacción hidrotermal. Los distintos procesos mencionados han transformado la mineralogía primaria (olivinos, piroxenos y feldespatos) en materiales secundarios como óxidos, oxihidróxidos, arcillas, sulfatos o carbonatos dependiendo del contexto petrológico y geoquímico de cada zona (Araña, 1979). 


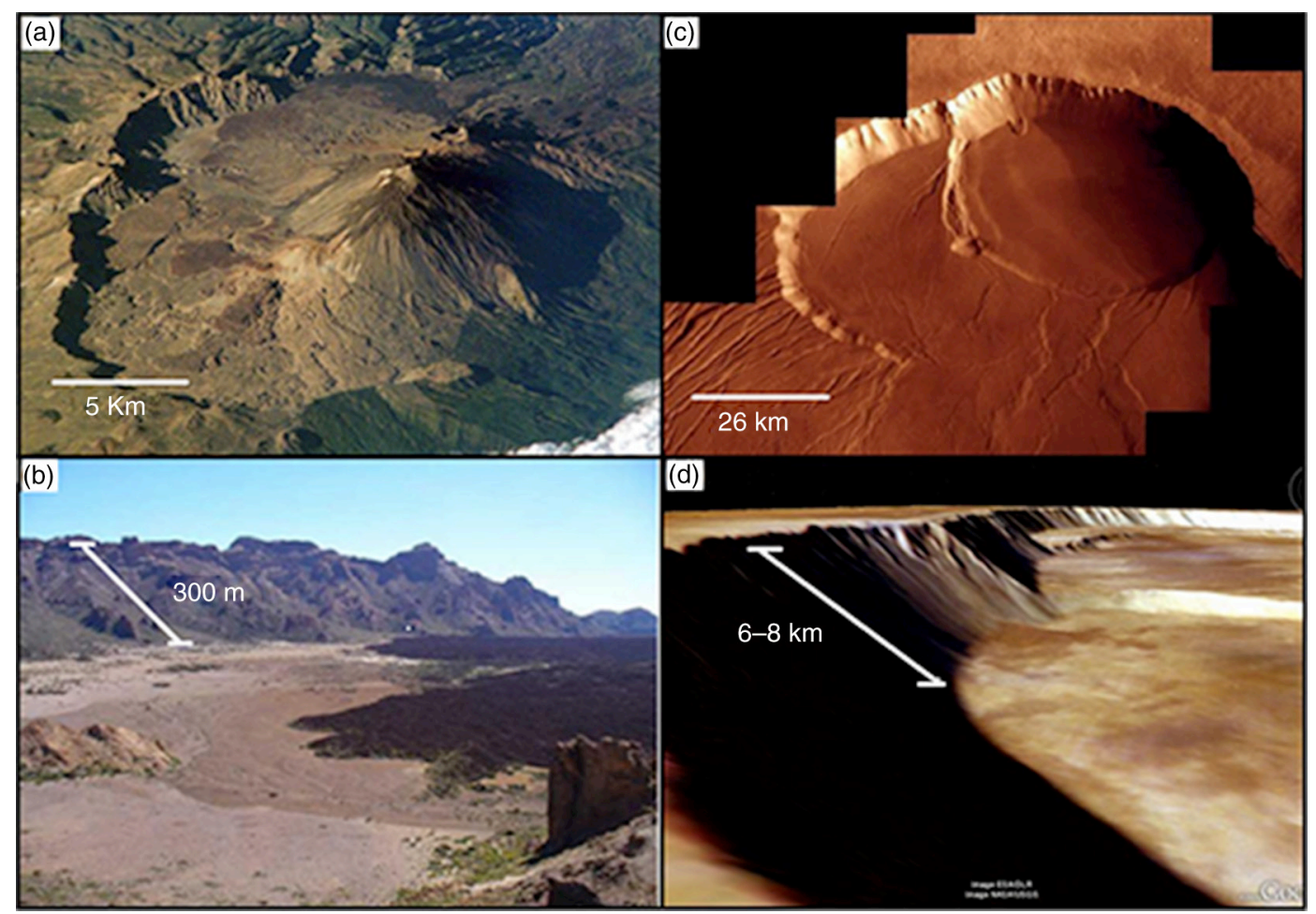

Fig. 1.-Imágenes comparativas de la morfología de la (a-b) Caldera de Las Cañadas y la (c-d) Caldera del Monte Olimpo (Créditos: NASA, 2000 y ESA, 2008).

Por otro lado, las distintas técnicas implicadas en misiones de exploración marciana como la difracción de rayos X, la espectroscopia IR y la espectroscopia Mössbauer han sido (y son) capaces de obtener información sobre los diferentes entornos geológicos en Marte (Klingelhofer et al., 2003; Ellery et al., 2003). Además, la espectroscopia Raman ha ganado gran aceptación en distintos campos de trabajo como las ciencias forenses, Biología o Arqueología. Del mismo modo, su desarrollo en el campo de la exploración planetaria ha crecido exponencialmente en los últimos años (Rull \& Martínez-Frías, 2006; Wang et al., 2003; Sharma et al., 2003). El uso de esta técnica en futuras misiones, como la misión ESA-ExoMars, requiere la caracterización previa de zonas y materiales en nuestro planeta, que puedan servir de análogos a las zonas que serán analizadas en Marte; de esta manera se conseguirá un mejor desarrollo y consolidación de los métodos de trabajo automatizado del instrumento Raman que irá a bordo de esta misión (Barnes et al., 2006; Rull et al., 2011).

En el presente trabajo se han estudiado algunos afloramientos de la isla de Tenerife especialmente interesantes por su potencial como posible análogo de Marte mediante el uso de instrumentación basada en la espectroscopia Raman, difracción de rayos X y espectroscopia Mössbauer. Esta selección incluye la Caldera de las Cañadas, las erupciones históricas (Lavas Negras y el Volcán del Chinyero) y la zona del Malpaís de Güimar.

\section{Descripción geológica de los afloramientos}

La Caldera de Las Cañadas presenta una geomorfología (Figuras 1 y 2) compleja, en la que se observan colapsos y depresiones, sobre cuya génesis se han planteado varias hipótesis (Martí et al., 1997; Martí \& Gudmundsson, 2000). En el Monte Olimpo de Marte se observa un colapso de $2,5 \mathrm{~km}$ en el interior de una caldera mayor, con un diámetro de $85 \times 60 \mathrm{~km}$ y con una elevación de al menos $2 \mathrm{~km}$ (Carr \& Greeley, 1980; Mouginis-Mark \& Robinson, 1992). Desde el punto de vista mineralógico-petrológico, la estructura tinerfeña presenta lavas fonolíticas, depósitos piroclásticos, ignimbritas o tefrifonolitas con una gran diversidad textural de plagioclasa intermedia, 


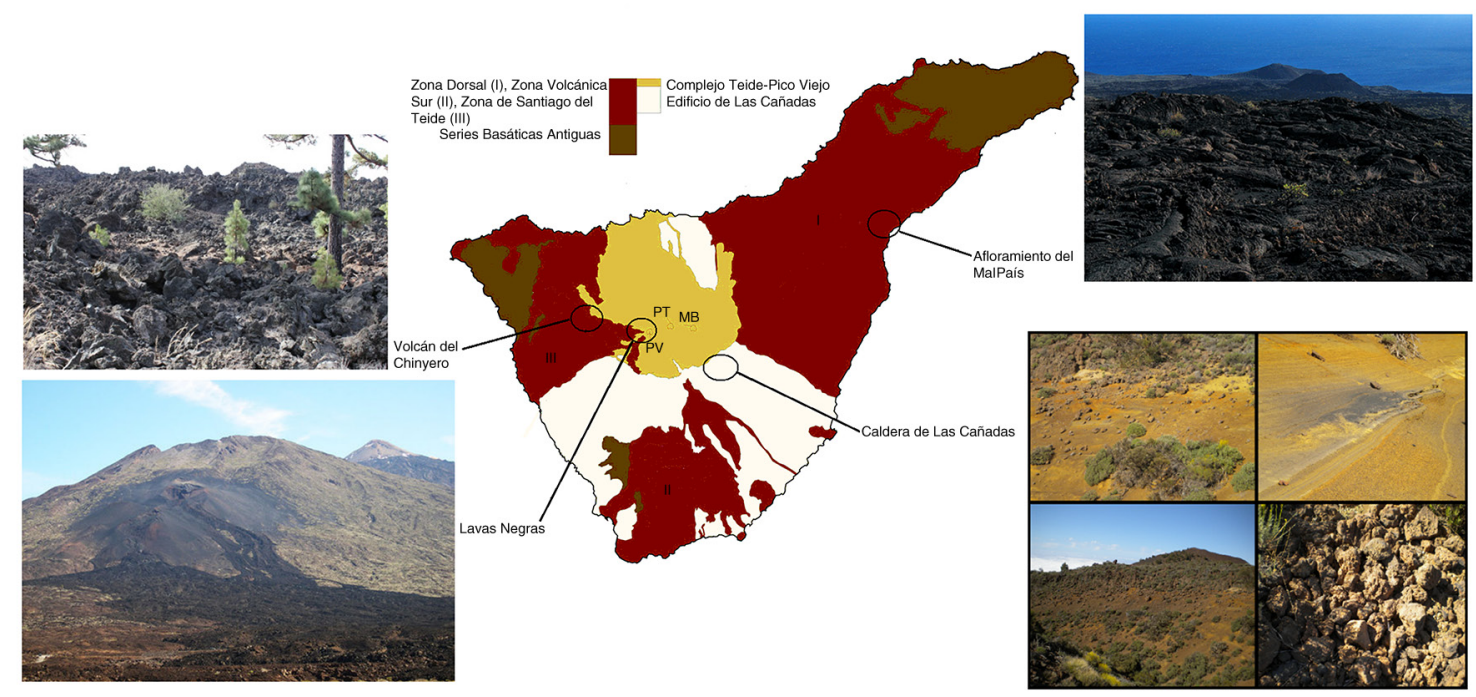

Fig. 2.-Mapa simplificado de la geología de Tenerife donde se ilustra la ubicación de las zonas seleccionadas y su entorno geológico. PT: Volcán del Teide, PV: Pico Viejo, MB: Montaña Blanca.

augita sódica, olivinos, clinopiroxenos y anfíbol entre otros (Rodríguez-Badiola \& Carracedo, 2008; Carr, 2007). Por otro lado, la zona de la Caldera de Las Cañadas es muy activa teniendo en consideración las emisiones volcánicas difusas debido a las anomalías en la distribución de la permeabilidad del material volcánico. Este hecho dio lugar a la aparición de una gran variedad de materiales secundarios de origen hidrotermal y relacionados con la emisión de gases, y con procesos de alteración subaéreos (Chevrier, 2007; Donoghue \& Troll, 2008; Rodríguez-Badiola \& Carracedo, 2008).

Las erupciones históricas (Lavas Negras y el volcán del Chinyero) datan de hace aproximadamente 500 años, estando perfectamente registradas en los libros históricos de la isla de Tenerife (Romero et al., 1986). Lavas Negras o Narices del Teide es una de las últimas erupciones ocurridas en la isla y se encuentra ubicada en el edificio central de la Caldera las Cañadas (gran estructura volcánica que conforma el sector central de Tenerife) que limita en un lateral con el sistema Teide-Pico Viejo (Figura 2). El volcán entró en erupción en 1798 creando 9 bocas que dieron lugar a varias coladas que se extienden por el sector sur de la caldera (Romero et al., 1986). Las bocas más elevadas emitieron fundamentalmente gases, las intermedias piroclastos, y las más bajas coladas de lava. Este tipo de comportamiento es muy común en los volcanes que tienen más de una boca eruptiva. La matriz de las lavas es de carácter vítreo con feldespatos alcalinos, además de contener numerosos fenocristales de clinopiroxenos y óxidos (Rodríguez-Badiola \& Carracedo, 2008). Por otro lado, el volcán del Chinyero se encuentra en una zona denominada dorsal Noroeste, en conjunto con otros volcanes como el Volcán de las Cuevitas o el Volcán de Boca Cangrejo, entró en erupción en 1909 dentro del municipio de Santiago del Teide. Los materiales de este entorno volcánico son íntegramente basálticos constituidos por conos, lavas y malpaís que se establecen a modo de islotes (Rodríguez-Badiola \& Carracedo, 2008). Desde el punto de vista petrológico se trata de un basalto constituido por fenocristales de augita diopsídica, cristales de olivino, cristales de feldespatos y plagioclasas idiomorfas (Rodríguez-Badiola \& Carracedo, 2008).

El afloramiento de Malpaís de Güimar es un conjunto volcánico reciente con una edad global menor a los diez mil años, constituido por un gran cono y un campo lávico asociado que se extiende hasta la costa (Figura 2). Los materiales de la zona son de naturaleza basáltica, aunque hay pequeños enclaves basálticos más antiguos, a modo de islote, que presentan una edad de diez mil años y no fueron cubiertos por la erupción más temprana de Montaña Grande (Risueño-Díaz, 2005; Romero et al., 1986). Geomorfológicamente, las coladas y los enclaves están agrupados en distintas unidades estratigráficas 
que se han sucedido a lo largo del tiempo. Los materiales que más abundan son una gran acumulación de piroclastos de diferente tamaño, desde finas cenizas pasando por lapillis, escorias y bombas volcánicas. Las coladas son lava tipo "aa" formando el denominado "malpaís", con zonas de lava pahoehoe, de aspecto más uniforme y donde se aprecian las evidencias de una actividad efusiva debido a los episodios de carácter explosivo (Risueño-Díaz, 2005; Romero et al., 1986). Lo más destacable de la zona son los procesos de alteración hidrotermal, subaérea, submarina y la interacción de materiales antiguos con los fluidos volcánicos más recientes.

El interés de Tenerife en relación con Marte puede sugerirse tanto en relación con similitudes de tipo geomorfológico como en relación con la diversidad petrológica y geoquímica de los conjuntos volcánicos en sus distintas zonas y episodios de formación y posterior alteración (Bish et al., 2013). En Marte, las rocas identificadas hasta la fecha son fundamentalmente, aunque no solo, de tipo basáltico (Christensen et al., 2003; McSween et al., 2009; Agee et al., 2013; Stolper et al., 2013). Por otro lado, aunque en el pasado no se habían encontrado evidencias de reciclamiento del manto marciano para producir materiales ígneos más evolucionados como el granito (Carr, 2007), estudios recientes sugieren una mayor complejidad, con evidencias que sí apuntan a la posible existencia de granitos (Wray et al., 2013). De esta manera, la evolución de los materiales ígneos marcianos y los procesos ocurridos en la geología marciana primitiva se podría entender si se adquiere un mejor conocimiento de las proporciones minerales, composiciones y texturas en los materiales de erupciones jóvenes de entornos volcánicos en la Tierra (Bish et al., 2013; Chevrier, 2007; Christensen et al., 2003; Horgan, 2013). De igual modo, los procesos de alteración hidrotermal como consecuencia de la interacción de los fluidos con los materiales volcánicos son de gran relevancia, ya que son capaces de generar minerales secundarios como óxidos y oxihidróxidos (Bustillo \& Martínez-Frías, 2003; Rodríguez-Losada et al., 2000). Un ejemplo de estos procesos en el ámbito de la geología marciana es el caso del cráter Gusev, donde se ha detectado abundancia de olivino, y también de materiales de alteración (Schmidt et al., 2009; Mustard et al., 2008).

\section{Muestreo y metodología}

El muestreo se llevó a cabo en varias campañas durante 2010 y 2011. Para ello, se contó con el apoyo de las autoridades del Parque y del personal del Museo de la Naturaleza y El Hombre y se siguieron en todo momento los protocolos establecidos, intentando integrar un conjunto de materiales frescos y alterados que fueran representativos para la realización de los análisis (Tabla 1).

Los análisis geoquímicos y mineralógicos mediante espectroscopia Raman se llevaron a cabo en la Unidad Asociada al Centro de Astrobiología de la Universidad de Valladolid mediante equipos comerciales. Los espectros obtenidos se compararon con los de la propia base de datos y muestras estándares. La instrumentación micro-Raman usada está compuesta por: un Láser He-Ne de $632.8 \mathrm{~nm}$, de $35 \mathrm{~mW}$ de potencia modelo LSRP-3501 Research ElectroOptics; un espectrógrafo Kaiser modelo HoloSpec f/1.8i con una red de difracción fijada para $633 \mathrm{~nm}$, trabajando en la zona espectral de $200-3800 \mathrm{~cm}^{-1}$ con una resolución máxima de $4 \mathrm{~cm}^{-1}$; un detector Andor CCD modelo Idus DV420A-OE-130 con una resolución $1024 \times 128$ de pixel con un tamaño de pixel de $26 \times 26 \mu \mathrm{m}$; una pletina motorizada en $3 \mathrm{D}$ : Prior Proscan II modelo H3V04 con posibilidad de programación automática; un microscopio Nikon modelo Eclipse E600 con posibilidad de magnificación de $10 \times, 20 \times, 50 \times$ y 100×; Cabezal Raman JobinYvon $633 \mathrm{~nm}$, UMS type; y una cámara CCD de control: cámara CCD para microscopio JVC modelo TK-C1381EG PAL con una resolución de 768×576.

Los sistemas de difracción de rayos X para el análisis de las muestras se encuentran disponibles en la Universidad de Valladolid y en la Unidad Asociada al Centro de Astrobiología de la Universidad de Valladolid. Se ha utilizado un sistema PANalytical Philips PW1710 equipado con un ánodo de $\mathrm{Cu}$, rendija automática divergente y monocromador de grafito. Las condiciones experimentales fueron las siguientes: radiación $\mathrm{CuK} \alpha, \lambda=0,154 \mathrm{~nm}$, filtro de $\mathrm{Ni}$, porta muestras de $\mathrm{Al}$, tensión del generador $40 \mathrm{KV}$, corriente del generador $30 \mathrm{~mA}$, relación de intensidades $\left(\alpha_{2} / \alpha_{1}\right)$ de 0.500 y análisis $(2 \theta)$ de $5^{\circ}$ a $70^{\circ}$. La asignación de los máximos de difracción se realizó mediante comparación con los ficheros JCPDS. Por otro, se recurrió también a un sistema de difracción portátil basado en 
Tabla 1.-Resumen de muestreos, ubicación geográfica (GPS), catalogación y análisis de espectroscopia Raman, DRX y Mössbauer por muestra. La mayoría de las muestras fueron analizadas en la Unidad Asociada, solo las muestras de mayor interés fueron examinadas mediante espectroscopia Mössbauer en la Universidad de Mainz

TNFC1

$\left(28^{\circ} 18^{\prime} 02^{\prime \prime} \mathrm{N} ; 16^{\circ} 33^{\prime} 58^{\prime \prime} \mathrm{O}\right)$

TNFC2

$\left(28^{\circ} 18^{\prime} 06^{\prime \prime} \mathrm{N} ; 16^{\circ} 33^{\prime} 56^{\prime \prime} \mathrm{O}\right)$

TNFC3

(281' $\left.18^{\prime \prime} \mathrm{W} ; 16^{\circ} 33^{\prime} 55^{\prime \prime} \mathrm{O}\right)$

TNFC4

$\left(28^{\circ} 18^{\prime} 16^{\prime \prime} \mathrm{N} ; 16^{\circ} 33^{\prime} 55^{\prime \prime O}\right)$

TNFC5

$\left(28^{\circ} 18^{\prime} 02^{\prime \prime} \mathrm{N} ; 16^{\circ} 33^{\prime} 33^{\prime \prime} \mathrm{O}\right)$

TNFC6

$\left(28^{\circ} 18^{\prime} 06^{\prime \prime} \mathrm{N} ; 16^{\circ} 33^{\prime} 48^{\prime \prime} \mathrm{O}\right)$ Fotografías

Análisis Raman Análisis DRX Análisis Mössbauer

\section{Las Caldera de las Cañadas}
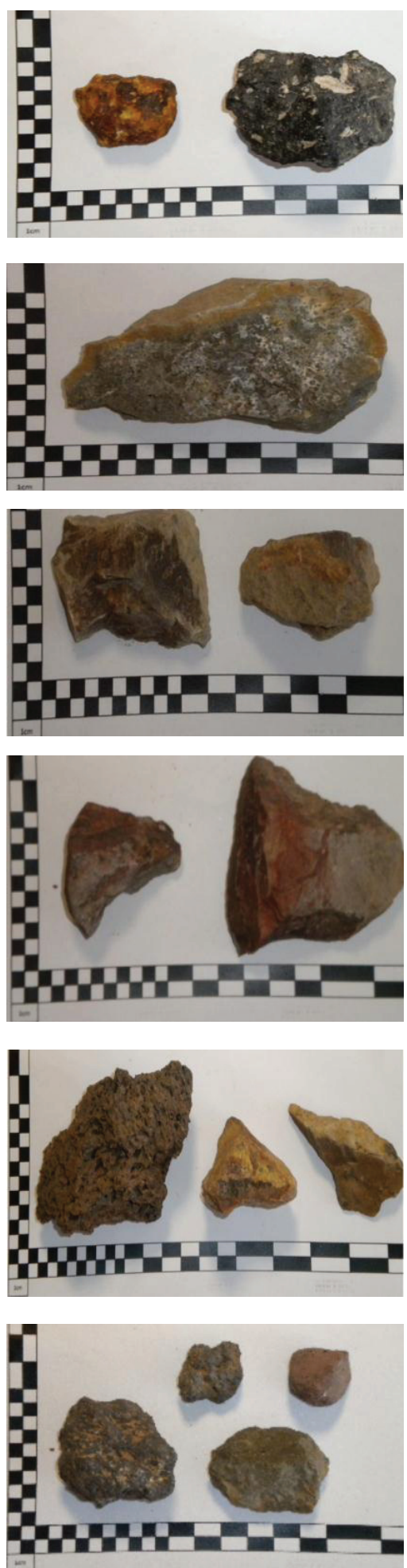

Magnetita Hematite

Goethita

Anatasa

Rutilo

Cuarzo

Augita

Diópsido

Ortoclasa

Anortoclasa

IImenita

Hidrotalcita

Albita

Magnetita

Hematite

Goethita

Anatasa

Cuarzo

Plagioclasas

Hematite

Anatasa

Cuarzo

Calcita

Apatito

Anortoclasa Albita

Goethita

Hematite

Anatasa

Rutilo

Cuarzo

Hidrotalcita

Augita

Diópsido

Actinolita

Forsterita

Oligoclasa

Magnetita, Hematite IImenita

Apatito

Oligoclasa

Ortoclasa
Cuarzo

Feldespatos

Illita

Moscovita

Diópsido

Piroxeno

Plagioclasa
Olivino

Silicato-Fe (Fase de Piroxeno)
Cuarzo

Piroxeno

Diópsido

Feldespatos

Albita

$\begin{array}{cc}\text { Arcillas } & \text { Magnetita } \\ \text { Piroxeno } & \text { Hematite } \\ \text { Feldespato } & \text { Goethita } \\ \text { Zeolita } & \text { Fe-OH }\end{array}$

Cuarzo

Diópsido

Forsterita

Moscovita

Hematite

Basanita

Diópsido

Feldespato

Hematite Albita
Fase $\mathrm{Fe}_{2} \mathrm{O}_{3}$ 
Table 1.-(Continued)

TNFC7

$\left(28^{\circ} 18^{\prime} 10^{\prime \prime} \mathrm{N} ; 16^{\circ} 39^{\prime} 42^{\prime \prime} \mathrm{E}\right)$

TNFC8

(2818'10"N;16³9'42"E)

TNFC9

(2818'06"N;16³3'28"E)

TNFC10

(281' $\left.15^{\prime \prime} \mathrm{N} ; 16^{\circ} 39^{\prime} 37^{\prime \prime} \mathrm{E}\right)$

TNFC11

(28॰18'14"N;16³3'30"E)

TNFLV

$\left(+28^{\circ} 13^{\prime} 50.57^{\prime \prime} \mathrm{N} ; 16^{\circ} 41^{\prime 2} 28^{\prime \prime O}\right)$

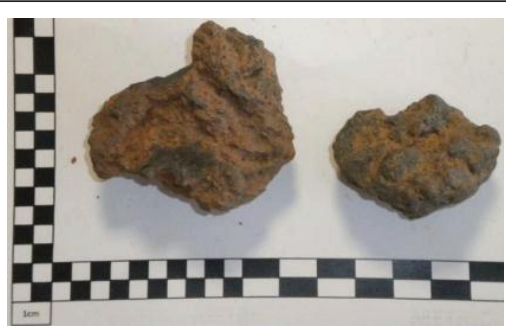

$\begin{array}{ccc}\text { Augita } & \text { Piroxeno } & \mathrm{Fase}^{\mathrm{Fe}_{2} \mathrm{O}_{3}} \\ \text { Diópsido } & \text { Diópsido } & \text { Magnetita } \\ \text { Forsterita } & \text { Feldespato } & \text { Olivino } \\ \text { Feldespato } & \text { Forsterita } & \text { Piroxeno }\end{array}$

Magnetita Hematite Augita

Diópsido

Actinolita

Forsterita

Magnetita Hematite

Augita

Diópsido

Actinolita

Forsterita

Monticellita

Albita

Hematite

Magnetita

Augita

Forsterita

Oligoclasa

Piroxeno

Diópsido

Plagioclasas
Óxido-Fe

Piroxeno

Olivino

Plagioclasas

Magnetita

Piroxeno

Forsterita

Plagioclasas
Hematite

Magnetita

Augita

Forsterita

Anortoclasa

Labradorita
Erupciones Históricas (volcán de Lavas Negras)

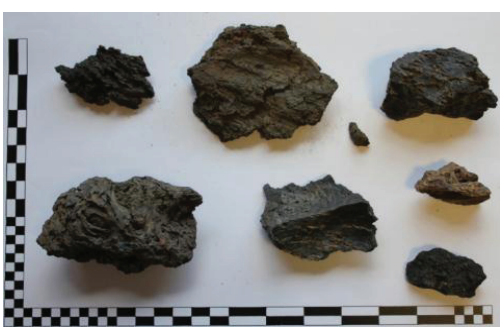

IImenita

Diópsido

Augita

Plagioclasas
Cuarzo

Piroxeno

Diópsido

Plagioclasas
Magnetita

Olivino

Piroxeno 


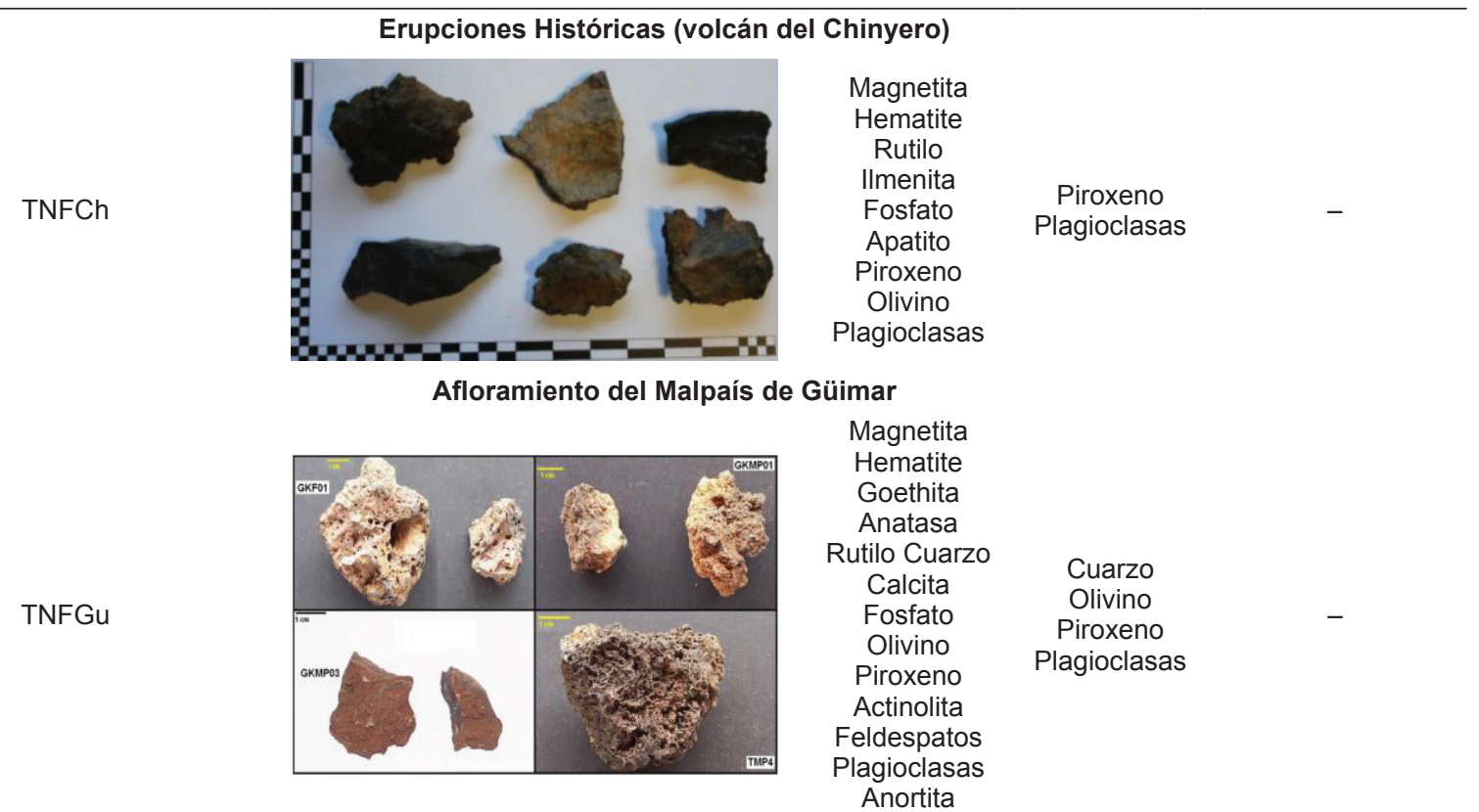

el CheMin TERRA-MSL actualmente a bordo en la Misión NASA-MSL-Curiosity. El instrumento fue diseñado para DRX-FRX y está compuesto por un detector $1024 \times 256$, una cámara CCD para DRX en $2 \mathrm{D}$ refrigerada mediante una celda peltier y un detector (Mn Ka) de $230 \mathrm{eV}$ para FRX. Además cuenta con una fuente de cobalto para rayos $\mathrm{X}$ de $30 \mathrm{Kv}-300 \mathrm{uA}$ (Bish, et al., 2006; Stalport, et al., 2012).

Los análisis mineralógicos mediante espectroscopia Mössbauer se realizaron en la Universidad de Johannes Gutenberg (Mainz, Alemania) mediante un sistema gemelo al usado en la mission NASA-MER Spirit y Opportunity. El sistema MIMOS II (Miniaturized Mössbauer Spectrometer) es un sistema de Mössbauer con arquitectura retro-dispersada desarrollado para exploración espacial para evitar la preparación de muestras. El diseño es el gemelo al modelo del laboratorio Athenas, de 400 gramos, con un consumo de $1 \mathrm{~W}$ y dimensiones de $50 \times 50 \times 90 \mathrm{~mm}$. Con respecto a la fuente, se utilizó una fuente de $\mathrm{Co}_{57} / \mathrm{Rh}$ con una intensidad de $50 \mathrm{mCi}$. Las medidas se realizaron a temperatura ambiente (Klingelhofer et al., 2010).

\section{Resultado y discusión}

En la Tabla 2 y Figuras 3-8 se resume la mineralogía detectada en las tres zonas a partir de los análisis realizados. Los materiales se han clasificado según las especies minerales y además de manera comparativa para las tres zonas.

\section{Espectroscopia Raman}

Mediante espectroscopia Raman se han detectado la gran mayoría de las especies minerales de carácter primario y también las de carácter secundario identificadas en trabajos previos (Rodriguez-Badiola \& Carracedo, 2008; Carracedo, 1975; Thirlwall et al., 2000; Carr, 2007; Romero et al., 1986; Risueño-Diaz, 2005).

Los olivinos en las tres zonas se corresponden a forsterita $\left(\mathrm{Mg}_{2} \mathrm{SiO}_{4}\right)$, los cuales son fácilmente identificables mediante las vibraciones principales a $820 \mathrm{y}$ $850 \mathrm{~cm}^{-1}$ (Kuebler et al., 2006). Aunque de forma puntual, en algunas muestras de las Cañadas se han podido encontrar otras especies olivínicas como monticellita $\left(\mathrm{CaMgSiO}_{4}\right)$. Kuebler et al. desarrollaron un método de análisis para diferenciar el tipo de olivino mediante las concentraciones relativas de los cationes $(\mathrm{Fe}, \mathrm{Mg})$ en función de las distancias relativas entre vibraciones Raman del doblete a 820 y $850 \mathrm{~cm}^{-1}$ (Tabla 3). Los métodos se aplicaron en las muestras de las distintas zonas obteniéndose forsterita (Kuebler et al., 2006; Mourí \& Enami, 2008).

En el caso de los piroxenos ( $(\mathrm{Ca}, \mathrm{Mg}, \mathrm{Fe}, \mathrm{Mn}, \mathrm{Na})$ $\left.(\mathrm{Al}, \mathrm{Mg}, \mathrm{Fe}, \mathrm{Mn})(\mathrm{Si}, \mathrm{Al})_{2} \mathrm{O}_{6}\right)$, se han aplicado los 
Tabla 2.- Resumen de todas las especies minerales detectadas en cada una de las zonas mediante las tres técnicas utilizadas

\begin{tabular}{|c|c|c|c|c|c|c|c|c|}
\hline \multirow[b]{2}{*}{ Especies Minerales } & \multicolumn{3}{|c|}{$\begin{array}{l}\text { La Cañadas } \\
\text { (Figura 3) }\end{array}$} & \multicolumn{3}{|c|}{$\begin{array}{c}\text { Erupciones Históricas } \\
\text { (Figura 4) }\end{array}$} & \multicolumn{2}{|c|}{$\begin{array}{c}\text { Malpaís de } \\
\text { Güimar (Figura 5) }\end{array}$} \\
\hline & Raman & DRX & Mössbauer & Raman & DRX & Mössbauer & Raman & DRX \\
\hline \multicolumn{9}{|l|}{ Óxidos e hidróxidos } \\
\hline Magnetita $\left(\mathrm{Fe}_{3} \mathrm{O}_{4}\right)$ & $x$ & $x$ & $x$ & $x$ & $x$ & $x$ & $x$ & \\
\hline Hematite $\left(\alpha-\mathrm{Fe}_{2} \mathrm{O}_{3}\right)$ & $x$ & $x$ & $x$ & $x$ & $x$ & & $x$ & \\
\hline Goethita $(\alpha-\mathrm{FeO}(\mathrm{OH}))$ & $x$ & $x$ & $x$ & & $x$ & & $x$ & \\
\hline Anatasa $\left(\mathrm{TiO}_{2}\right)$ & $x$ & & & $x$ & & & $x$ & \\
\hline Rutilo $\left(\mathrm{TiO}_{2}\right)$ & $x$ & & & $x$ & & & $x$ & \\
\hline Cuarzo $\left(\mathrm{SiO}_{2}\right)$ & $x$ & $x$ & & $x$ & $x$ & & $x$ & \\
\hline Sílice $\left(\mathrm{SiO}_{2}\right)$ & $x$ & & & $x$ & & & $x$ & \\
\hline Ilmenita $\left(\mathrm{FeTiO}_{3}\right)$ & $x$ & & & $x$ & & & $x$ & \\
\hline \multicolumn{9}{|l|}{ Carbonatos } \\
\hline Calcita $\left(\mathrm{CaCO}_{3}\right)$ & $x$ & & & $x$ & & & $x$ & \\
\hline Hidrotalcita $\left(\mathrm{Mg}_{6} \mathrm{Al}_{2}\left(\mathrm{CO}_{3}\right)(\mathrm{OH})_{16} \cdot 4\left(\mathrm{H}_{2} \mathrm{O}\right)\right.$ & $x$ & & & & & & & \\
\hline \multicolumn{9}{|l|}{ Fosfatos } \\
\hline Apatito $\left(\mathrm{Ca}_{5}\left(\mathrm{PO}_{4}\right)_{3}(\mathrm{~F}, \mathrm{Cl}, \mathrm{OH})\right)$ & $x$ & & & $\mathrm{x}$ & & & $x$ & \\
\hline \multicolumn{9}{|l|}{ Silicatos } \\
\hline \multicolumn{9}{|l|}{ Inosilicatos } \\
\hline Piroxenos & $x$ & $x$ & $x$ & $x$ & $x$ & $x$ & $x$ & $x$ \\
\hline Diópsido $\left(\mathrm{MgCaSi}_{2} \mathrm{O}_{6}\right)$ & $x$ & $x$ & $x$ & $\mathrm{x}$ & $\mathrm{x}$ & $x$ & $x$ & $x$ \\
\hline Augita $\left((\mathrm{Ca}, \mathrm{Mg}, \mathrm{Fe})_{2}(\mathrm{Si}, \mathrm{Al})_{2} \mathrm{O}_{6}\right)$ & $x$ & $x$ & $x$ & $x$ & $x$ & $x$ & $x$ & $x$ \\
\hline Anfíboles & $x$ & & & & & & $x$ & \\
\hline Actinolita $\left(\mathrm{Ca}_{2}\left(\mathrm{Mg}, \mathrm{Fe}^{2+}\right)_{5} \mathrm{Si}_{8} \mathrm{O}_{22}(\mathrm{OH})_{2}\right)$ & $x$ & & & & & & $x$ & \\
\hline \multicolumn{9}{|l|}{ Nesosilicatos } \\
\hline Olivinos & $x$ & $x$ & $x$ & $x$ & $x$ & $x$ & $x$ & $x$ \\
\hline Forsterita $\left((\mathrm{Fe}, \mathrm{Mg})_{2} \mathrm{SiO}_{4}\right)$ & $x$ & $x$ & & & & & $x$ & $x$ \\
\hline Monticellita $\left(\mathrm{CaMgSiO}_{4}\right)$ & $x$ & & & & & & & \\
\hline \multicolumn{9}{|l|}{ Tectosilicatos } \\
\hline Feldespatos & $x$ & $x$ & & $x$ & $x$ & & $x$ & \\
\hline Anortoclasa $\left((\mathrm{Na}, \mathrm{K}) \mathrm{AISi}_{3} \mathrm{O}_{8}\right)$ & $x$ & $x$ & & $x$ & & & $x$ & \\
\hline Ortoclasa y Microclina $\left(\mathrm{KAISi}_{3} \mathrm{O}_{8}\right)$ & $x$ & $x$ & & & $\mathrm{X}$ & & $x$ & \\
\hline Sanidina $\left((\mathrm{K}, \mathrm{Na})(\mathrm{Si}, \mathrm{Al})_{4} \mathrm{O}_{8}\right)$ & $x$ & $x$ & & $\mathrm{X}$ & $x$ & & $x$ & \\
\hline Anortita $\left(\mathrm{CaAl}_{2} \mathrm{Si}_{2} \mathrm{O}_{8}\right)$ & $x$ & $x$ & & $\mathrm{x}$ & $x$ & & $x$ & \\
\hline Albita $\left(\mathrm{NaAISi}_{3} \mathrm{O}_{8}\right)$ & $x$ & $x$ & & $x$ & $x$ & & $x$ & \\
\hline Oligoclasa y Andesina $\left((\mathrm{Na}, \mathrm{Ca})(\mathrm{Si}, \mathrm{Al})_{4} \mathrm{O}_{8}\right)$ & $x$ & $x$ & & $x$ & $x$ & & $x$ & \\
\hline Labradorita $\left((\mathrm{Ca}, \mathrm{Na})(\mathrm{Si}, \mathrm{Al})_{4} \mathrm{O}_{8}\right)$ & $x$ & $x$ & & $x$ & $x$ & & $x$ & \\
\hline Zeolitas & $x$ & & & $x$ & & & & \\
\hline Phillipsita $\left((\mathrm{Ca}, \mathrm{K}, \mathrm{Na})_{6}\left(\mathrm{Si}_{10} \mathrm{Al}_{6}\right) \mathrm{O}_{32} 12 \mathrm{H}_{2} \mathrm{O}\right)$ & $x$ & & & & & & & \\
\hline Analcima $\left(\mathrm{Na}\left(\mathrm{AISi}_{2} \mathrm{O}_{6}\right) \cdot \mathrm{H}_{2} \mathrm{O}\right)$ & $x$ & & & & & & $x$ & \\
\hline \multicolumn{9}{|l|}{ Filosilicatos } \\
\hline Arcillas & & $x$ & & & & & & \\
\hline Caolinita $\left(\mathrm{Al}_{2} \mathrm{Si}_{2} \mathrm{O}_{5}(\mathrm{OH})_{4}\right)$ & & $x$ & & & & & & \\
\hline Moscovita $\left(\mathrm{KAl}_{2}\left(\mathrm{AISi}_{3} \mathrm{O}_{10}\right)(\mathrm{OH})_{2}\right)$ & & $\mathrm{x}$ & & & & & & \\
\hline Illita $\left(\mathrm{K}, \mathrm{H}_{3} \mathrm{O}\right)(\mathrm{Al}, \mathrm{Mg}, \mathrm{Fe})_{2}(\mathrm{Si}, \mathrm{Al})_{4} \mathrm{O}_{10}(\mathrm{OH}),\left(\mathrm{H}_{2} \mathrm{O}\right)$ & & $x$ & & & & & & \\
\hline \multicolumn{9}{|l|}{ Material Carbonáceo } \\
\hline Grafito & $\mathrm{X}$ & & & $\mathrm{X}$ & & & $x$ & \\
\hline
\end{tabular}




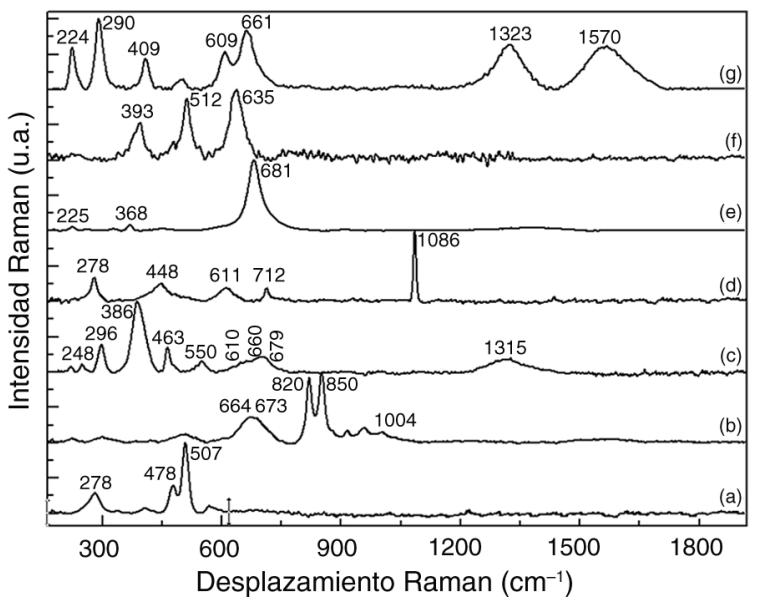

Fig. 3.-Espectros Raman de especies minerales detectadas en la Caldera de Las Cañadas: (a) Plagioclasas, (b) Forsterita+Piroxeno +Actinolita, (c) Goethita + Hematite + Cuarzo, (d) Rutilo + Calcita, (e) Ilmenita, (f) Anatasa y (g) Hematite+Magnetita + Carbón/Grafito.

métodos de análisis cuantitativos mediante espectroscopia Raman desarrollados por Huang et al. (2000) y Wang et al. (2001) para lograr una correcta identificación de los mismos (Figura 6 y Tabla 4). Las especies minerales son diópsido $\left(\mathrm{CaMgSi}_{2} \mathrm{O}_{6}\right)$ y augita $\left((\mathrm{Ca}, \mathrm{Mg}, \mathrm{Fe})_{2}(\mathrm{Si}, \mathrm{Al})_{2} \mathrm{O}_{6}\right)$ en la mayoría de las zonas y de los casos, fácilmente identificables mediante las vibraciones típicas a 660 y $1010 \mathrm{~cm}^{-1}$ debidas al modo stretching $\mathrm{Si}-\mathrm{O}_{b r}$ y $\mathrm{Si}-\mathrm{O}_{n b r}$, aunque también hay otras vibraciones más intensas que han sido estudiadas (Wang et al., 2001).

La caracterización Raman de los feldespatos se realizó mediante los métodos realizados por Freeman et al. (2008) a través de las vibraciones principales doblete/triplete a 460/470, 480 y $505 / 510 \mathrm{~cm}^{-1}$ (Zoltai \& Scout, 1984). Las muestras presentaban especies muy variadas de feldespatos y plagioclasas, las cuales cambian dependiendo de las muestras.

Los óxidos dependiendo del elemento (Fe, Ti, Si) tendrán una estructura, y por lo tanto, unas vibraciones Raman diferentes. Las identificaciones de las especies de óxidos/hidróxidos de $\mathrm{Fe}$ se han realizado en base a las vibraciones de los grupos activos en Raman para cada mineral. La hematite $\left(\alpha-\mathrm{Fe}_{2} \mathrm{O}_{3}\right)$ posee las vibraciones principales a 220295,607 y el magnón a $1315 \mathrm{~cm}^{-1}$ (Rull et al., 2007); en cambio la magnetita $\left(\mathrm{Fe}^{2+}\left(\mathrm{Fe}^{3+}\right)_{2} \mathrm{O}_{4}\right)$ presenta sus vibraciones más intensas en la zona de 670 y $550 \mathrm{~cm}^{-1}$ (Jubb \&
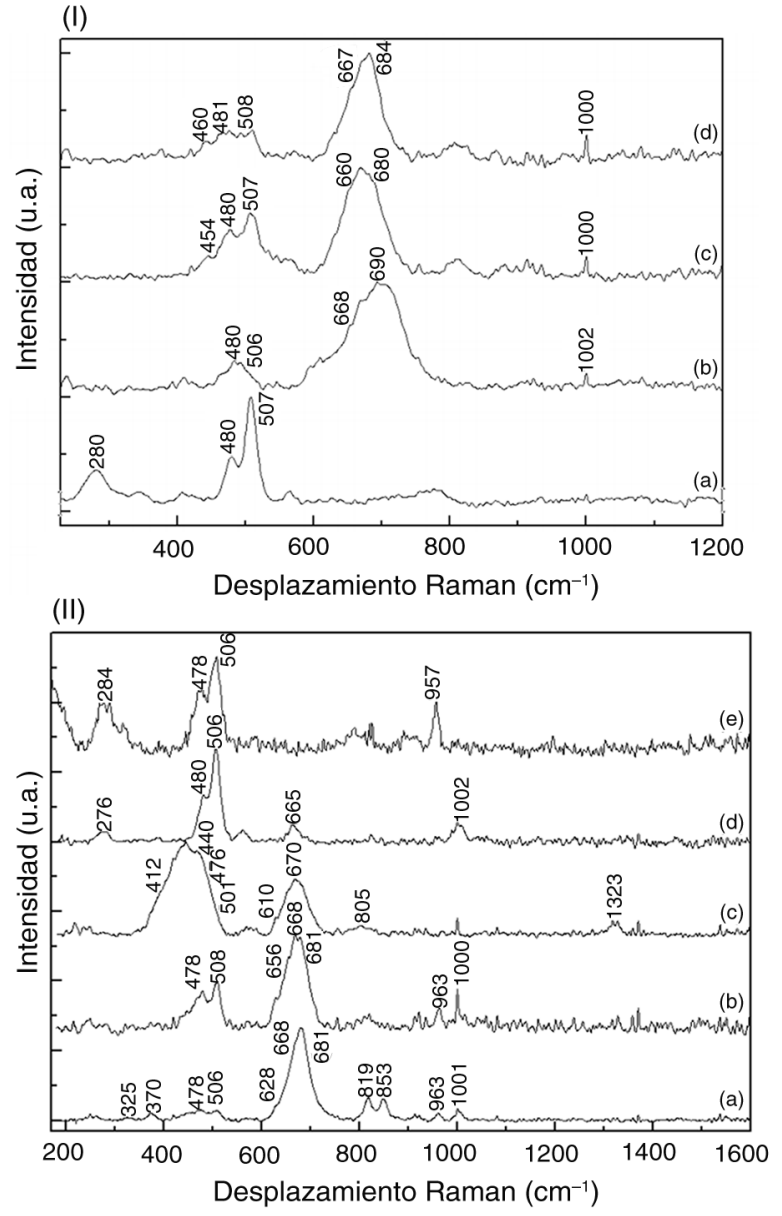

Fig. 4.-(I) Espectros Raman de especies minerales detectadas en la zona de Lavas Negras: (a) Plagioclasa, (b) Plagioclasa + Piroxeno + Ilmenita, (c) Plagioclasa +Enstatita y (d) Plagioclasa +Piroxeno + IImenita. (II) Espectros Raman de especies minerales detectadas en la zona del volcán del Chinyero: (a) Piroxeno + Olivino + IImenita + Plagioclasa + Fosfato (b) Piroxeno + Apatito + IImenita + Plagioclasa + Magnetita (c) Hematite + Magnetita + Plagioclasa + Rutilo (d) Plagiolacasa + Piroxeno (e) Plagioclasa + Fosfato.

Allen, 2010; Rull et al., 2007). Por último la goethita $(\alpha-\mathrm{FeO}(\mathrm{OH}))$ presenta sus vibraciones a 248,296 , 386, 550 y $682 \mathrm{~cm}^{-1}$. En el caso de los óxidos de titanio, la anatasa $\left(\mathrm{TiO}_{2}\right)$ tiene vibraciones Raman a 393,512 y $635 \mathrm{~cm}^{-1}$ (Sekiya et al., 2001) y el rutilo $\left(\mathrm{TiO}_{2}\right)$ presenta vibraciones muy intensas a 235,448 y $810 \mathrm{~cm}^{-1}$ (Krisshnamurti, 1962).

El cuarzo $\left(\mathrm{SiO}_{2}\right)$ posee una vibración muy intensa a $463 \mathrm{~cm}^{-1}$, y otras vibraciones menos intensas a 355 y $205 \mathrm{~cm}^{-1}$ que en algunos casos no fueron detectadas debido al solapamiento de otras especies minerales 


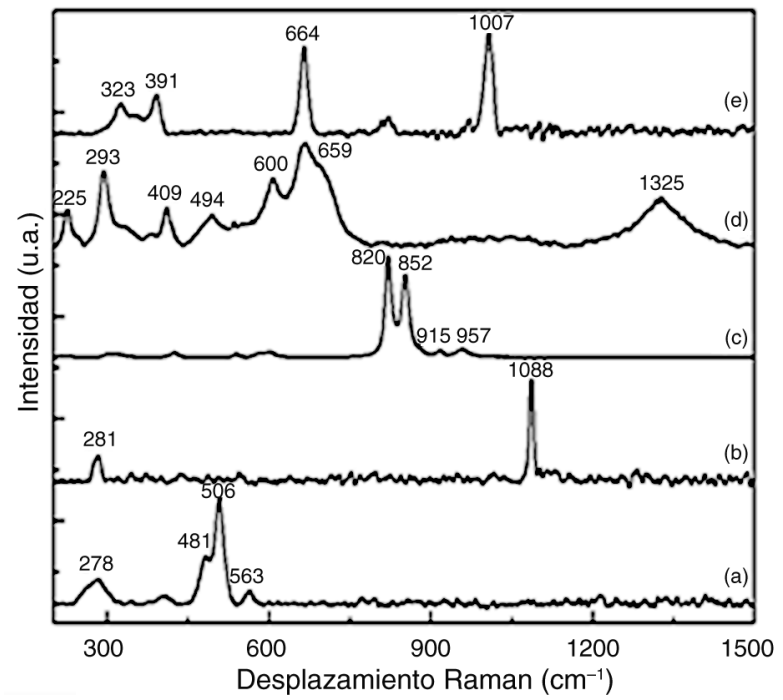

Fig. 5.-Espectros Raman de especies minerales detectadas en la zona del Malpaís de Güimar: (a) Plagioclasa, (b) Carbonato, (c) Olivino, (d) Hematite + Magnetita, (e) Piroxeno.

(Dong Zu et al., 2011; Zotov \& Ebbsjo, 1999). E1 último de los óxidos encontrado en las zonas es la ilmenita $\left((\mathrm{Fe}, \mathrm{Ti}) \mathrm{O}_{3}\right)$ donde sus bandas principales están ubicadas a 681,368 y $225 \mathrm{~cm}^{-1}$ (Chopelas, 1999; Okada et al., 2008).
El único carbonato detectado es calcita y podría deberse a procesos de alteración superficial, aunque también podría corresponderse a los de tipo volcánico debido a la interacción con fluidos hidrotermales. En el caso de la calcita $\left(\mathrm{CaCO}_{3}\right)$, considerando las vibraciones principales a 1086,712 y $280 \mathrm{~cm}^{-1}$ y realizando una comparativa entre distancias relativas de bandas e intensidades relativas, se confirma el origen volcánico de los mismos (Rull \& MartínezFrías, 2003). También se ha detectado hidrotalcita $\left(\left(\mathrm{Mg}_{6} \mathrm{Al}_{2}\left(\mathrm{CO}_{3}\right)(\mathrm{OH})_{16} \cdot 4\left(\mathrm{H}_{2} \mathrm{O}\right)\right)\right.$; posee las vibraciones más intensas a 1060 y $1113 \mathrm{~cm}^{-1}$, y otras más débiles debidas a vibraciones Al-OH a 992 y $540 \mathrm{~cm}^{-1}$ (Kloprogge et al., 2002).

Entre los materiales accesorios, destacan los fosfatos de tipo apatito, identificados mediante las bandas ubicadas a 1017,960 y $563 \mathrm{~cm}^{-1}$ (Zattina et al., 2007).

El anfíbol detectado con más frecuencia en las zonas propuestas como análogos terrestres es la actinolita $\left(\mathrm{Ca}_{2}\left(\mathrm{Mg}, \mathrm{Fe}^{2+}\right)_{5} \mathrm{Si}_{8} \mathrm{O}_{22}(\mathrm{OH})_{2}\right)$, especialmente en las Caldera de las Cañadas y en los afloramientos del Malpaís de Güimar. Las bandas de esta especie mineral se encuentran ubicadas a 229, 391, 670 y $1050 \mathrm{~cm}^{-1}$ (Apopei \& Buzgar, 2010), aunque en la

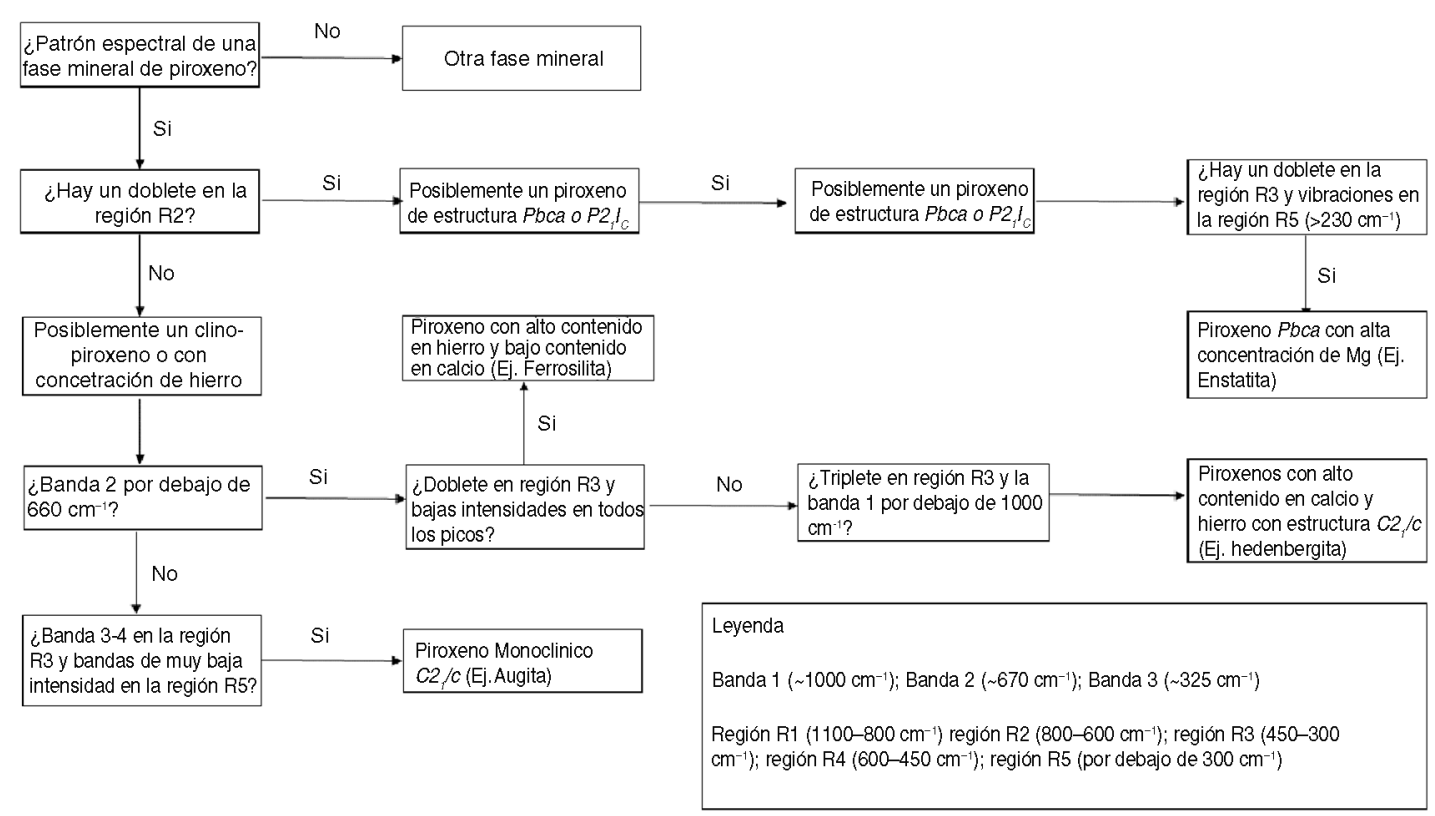

Fig. 6.-Diagrama de flujos para la identificación de piroxenos en los afloramientos estudiados de Tenerife. El diagrama de flujo se basa en la identificación de bandas Raman y su patrón desarrollado por Wang et al. (Wang et al., 2001). El diagrama ha sido traducido al español y además se añade la asignación de picos necesaria (Leyenda). 


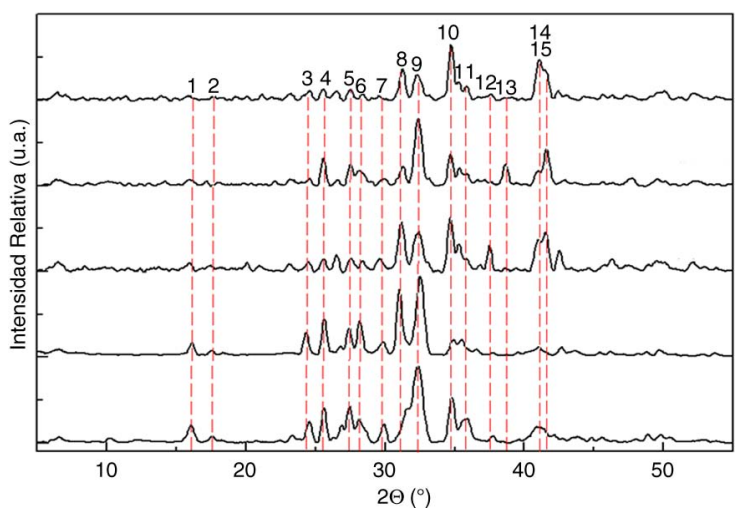

Fig. 7.-Difracción de Rayos X (Terra Che-Min) de algunas de las muestras recogidas en los distintos afloramientos. Asignación de Picos: Feldespatos: 1, 2, 3, 4, 5, 6, 7, 8, 9, 10, 11, 12, 14, 15; Piroxenos: 1, 3, 5, 7, 8, 9, 10, 11, 12, 14, 15; Óxidos (Cuarzo): 3, 8, 15; Olivinos: 5, 7, 12, 15; Óxidos (Hematite): 6, 13, 15; Arcillas (Moscovita): 4, 8, 9, 10, 14.

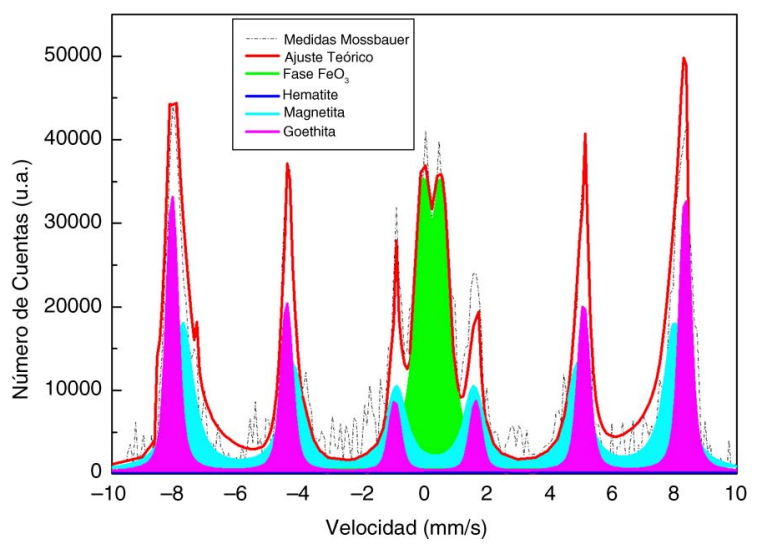

Fig. 8.-Espectro Mössbauer de la zona de La Caldera de Cañadas con la identificación de las especies obtenidas mediante ajustes numéricos de los patrones espectrales.

mayoría de los casos, se detectó en conjunto con otras bandas Raman solapadas correspondientes a otras especies minerales.

Entre los materiales de alteración se han encontrado tanto arcillas como zeolitas, que podrían tener distintos orígenes, tanto de carácter hidrotermal, como meteorización. La analcima $\left(\mathrm{Na}\left(\mathrm{Si}_{2} \mathrm{Al}\right) \mathrm{O}_{6} \cdot \mathrm{H}_{2} \mathrm{O}\right)$ presenta sus vibraciones principales a 384,480 y $1100 \mathrm{~cm}^{-1}$ (Hofmeister et al., 1999), claramente diferenciable de la moscovita $\left(\mathrm{KAl}_{2}\left(\mathrm{AlSi}_{3} \mathrm{O}_{10}\right)(\mathrm{OH})_{2}\right)$ con bandas intensas a 290, 406, 700 y $1100 \mathrm{~cm}^{-1}$ (Mckeown et al., 1999; Haley et al., 1982) y la caolinita a 640, 510, 333 y $258 \mathrm{~cm}^{-1}$ (Frost \& Kloprogge, 2001). Los materiales zeolíticos (phillipsitas) de la
Caldera de Las Cañadas se han detectado en zonas de circulación de fluidos o micro-fisuras.

\section{Difracción de rayos $X$}

Los resultados de DRX mostrados en la Tabla 1 y 2 revelan que en su mayoría las matrices primarias están constituidas por piroxenos, olivinos y feldespatos coincidiendo con la literatura (Rodríguez-Badiola \& Carracedo, 2008; Marti et al., 1997; Marti \& Gudmundsson, 2000). En algunos casos se han podido identificar las especies secundarias mayoritarias, pero al trabajar con el método del polvo policristalino, algunas especies que se detectaron mediante Raman en zonas puntuales de las muestras se encuentran solapadas con las fases principales mayoritarias (Figura 7). Los análisis realizados entre los diferentes equipos no presentaron diferencias, esto indica las altas capacidades del instrumental In-situ-Terra en términos de identificación mineral.

\section{Espectroscopia Mössbauer}

Considerando los parámetros más importantes en la espectroscopia Mössbauer: los desplazamientos espectrales (Centershift), los desdoblamientos del cuadrupolo eléctrico (electric quadrupole splitting), la estructura hiperfina magnética (magnetic hyperfine field) y la anchura de las bandas (isomeric pattern) se pueden detectar las especies minerales con un alto contenido en $\mathrm{Fe}$ (Klingelhofer et al., 1992), aunque también se puede interpretar la estructura electrónica que poseen, la simetría molecular y los estados de oxidación (Fleischer, 2010). Tanto en la Tabla 5 como en la Figura 8 se muestran los análisis realizados en las muestras de mayor interés.

En el caso de las erupciones históricas, los afloramientos presentan una alta proporción de materiales basálticos incluyendo minerales olivínicos y piroxénicos. También se han observado especies minerales correspondientes a óxidos (magnetita, hematite y goethita), aunque algunas fases de óxidos de hierro presentan una gran anchura en las bandas (Lalla, 2014).

En el caso de la Caldera de las Cañadas, además de los olivinos y piroxenos, se han detectado más variedades de fases amorfas de óxidos que en las zonas de erupciones históricas. En las Cañadas el óxido 
Tabla 3.-Análisis de bandas de los olivinos en los distintos afloramientos a través de las vibraciones principales (Kuebler et al., 2006; Mourí \& Enami, 2008)

\begin{tabular}{|c|c|c|c|c|}
\hline Muestra & $\begin{array}{l}\text { Vibración a } \\
820 \mathrm{~cm}^{-1} \text { (DB1) }\end{array}$ & $\begin{array}{l}\text { Vibración a } \\
850 \mathrm{~cm}^{-1} \text { (DB2) }\end{array}$ & Ecuación (1) & Ecuación (2) \\
\hline \multicolumn{5}{|c|}{ Las Caldera de las Cañadas } \\
\hline TNFC5 & 820 & 850 & Forsterita $_{80}$ & Forsterita $_{75-80}$ \\
\hline TNFC7 & 823 & 847 & Forsterita $_{90}$ & Forsterita $_{75-90}$ \\
\hline TNFC8 & 821 & 851 & Forsterita $_{80-90}$ & Forsterita $_{80-85}$ \\
\hline TNFC9 & 819 & 849 & Forsterita $_{70}$ & Forsterita $_{75-80}$ \\
\hline TNFC10 & 821 & 853 & Forsterita $_{90}$ & Forsterita $_{85-90}$ \\
\hline TNFC11 & 820 & 849 & Forsterita $_{70-80}$ & Forsterita $_{70-80}$ \\
\hline \multicolumn{5}{|c|}{ Erupciones Históricas (volcán del Chinyero) } \\
\hline \multirow{2}{*}{ TNFCh } & 819 & 853 & Forsterita $60-70$ & Forsterita $_{80-90}$ \\
\hline & 821 & 849 & Forsterita $_{80}$ & Forsterita $_{70-90}$ \\
\hline \multicolumn{5}{|c|}{ Afloramiento del Malpaís de Güimar } \\
\hline \multirow{2}{*}{ TNGu } & 820 & 852 & Forsterita $_{90}$ & Forsterita $_{80-85}$ \\
\hline & 818 & 847 & Forsterita $40-60$ & Forsterita $_{60-65}$ \\
\hline $\begin{array}{l}\text { Ecuación de ajuste } 1 \\
\text { (Kuebler et al., 2006) }\end{array}$ & \multicolumn{4}{|c|}{$\begin{array}{c}\text { Fo(DB1, } \mathrm{DB} 2)=-206232.988995287+80.190397775029(\mathrm{DB} 1)+399.350231139156 \\
(\mathrm{DB} 2)-0.0424363912074934(\mathrm{DB} 1)^{2}-0.2357973451030880(\mathrm{DB} 2)^{2}\end{array}$} \\
\hline $\begin{array}{l}\text { Ecuación de ajuste } 2 \\
\text { (Mourí \& Enami, 2008) }\end{array}$ & \multicolumn{4}{|c|}{$\begin{array}{l}M g \#=-610.65+1.3981(\mathrm{DB} 2)-0.00079869(\mathrm{DB} 2)^{2} \\
M g \#=-3715.8+8.9889(\mathrm{DB} 1)-0.0054348(\mathrm{DB} 1)^{2}\end{array}$} \\
\hline
\end{tabular}

Tabla 4.-Análisis de bandas de los piroxenos en los distintos afloramientos a través de las vibraciones principales, solo se han considerado las bandas más intensas usadas a partir del diagrama de flujos desarrollado por Wang et al. (Figuras 5-6). Las ecuaciones de ajuste se basan para la serie DiópsidoHedenbergita teniendo en cuenta el contenido en $\mathrm{Fe}$

\begin{tabular}{lcccc}
\hline Muestra & $\begin{array}{c}\text { Vibración a } \\
1000 \mathrm{~cm}^{-1} \text { (DB1) }\end{array}$ & $\begin{array}{c}\text { Vibración a } \\
665 \mathrm{~cm}^{-1} \text { (DB2) }\end{array}$ & $\begin{array}{c}\text { Vibración a } \\
325 \mathrm{~cm}^{-1} \text { (DB3) }\end{array}$ & $\begin{array}{c}\text { Ecuación/ } \\
\text { Resultado }\end{array}$ \\
\hline TNFC1 & \multicolumn{4}{c}{ Las Caldera de las Cañadas } \\
TNFC5 & 1001 & 664 & 324 & Diópsido \\
TNFC7 & 1000 & 660 & 321 & Diópsido \\
TNFC9 & 1008 & 664 & - & Diópsido \\
TNFC10 & 1003 & 663 & 324 & Diópsido \\
& 1004 & 664 & - & Diópsido \\
TNFCh & Erupciones Históricas (volcán del Chinyero) & Diópsido \\
& 1002 & 665 & - & Diópsido \\
TNFL & 1001 & 668 & 325 & Diópsido \\
& Erupciones Históricas (volcán de Lavas Negras) & Diópsido \\
TNGu & 1000 & 667 & 323 & Diópsido \\
Ecuación de ajuste & 1002 & 668 & Diópsido \\
(Huang et al., 2000) & 1007 & Afloramiento del Malpaís de Güimar & 323 &
\end{tabular}

principal es la magnetita, aunque se han detectado también de manera abundante hematite y goethita (Lalla, 2014). En los análisis Raman y Mössbauer se pueden apreciar las variaciones en los valores de los óxidos y silicatos. Los estados de oxidación $\mathrm{Fe}^{3+}$ y Fe ${ }^{2+}$ están estrechamente relacionados con los 


\begin{tabular}{|c|c|c|c|}
\hline Muestra & IS (mm/s) & $\mathrm{QS}(\mathrm{mm} / \mathrm{s})$ & $\Gamma(\mathrm{mm} / \mathrm{s})$ \\
\hline \multicolumn{4}{|c|}{ TNFC1 (Las Caldera de las Cañadas) } \\
\hline Olivino & 0.90 & 2.90 & 0.15 \\
\hline Silicato (Piroxeno) & 0.24 & 3.4 & 0.11 \\
\hline \multicolumn{4}{|c|}{ TNFC4 (Las Caldera de las Cañadas) } \\
\hline Magnetita & 0.38 & 0.13 & 0.38 \\
\hline Hematite & 0.57 & 16.45 & 0.16 \\
\hline Goethita & 0.13 & 16.42 & 0.11 \\
\hline Fase $\mathrm{Fe}-\mathrm{OH}$ & 0.14 & 0.58 & 0.29 \\
\hline \multicolumn{4}{|c|}{ TNFC6 (Las Caldera de las Cañadas) } \\
\hline Hematite & 0.80 & 13.21 & 0.18 \\
\hline Fase de $\mathrm{Fe}_{2} \mathrm{O}_{3}$ & 0.15 & 0.46 & 0.20 \\
\hline \multicolumn{4}{|c|}{ TNFC7 (Las Caldera de las Cañadas) } \\
\hline Magnetita & 0.14 & 15.47 & 0.40 \\
\hline Fase de $\mathrm{Fe}_{2} \mathrm{O}_{3}$ & 0.16 & 0.68 & 0.27 \\
\hline Olivino & 0.81 & 2.45 & 0.27 \\
\hline Fase de Piroxeno & 0.67 & 1.97 & 0.38 \\
\hline \multicolumn{4}{|c|}{ TNFL (Volcán de Lavas Negras) } \\
\hline Magnetita & 0.10 & 15.75 & 0.40 \\
\hline Olivino & 0.80 & 2.41 & 0.31 \\
\hline Piroxeno & 0.62 & 1.10 & 0.42 \\
\hline Fase de $\mathrm{Fe}_{2} \mathrm{O}_{3}$ & 0.15 & 0.68 & 0.24 \\
\hline
\end{tabular}

desplazamientos isoméricos (Stevens et al., 2005). De esta manera, las variaciones de posición y anchura de las bandas están directamente relacionadas con la energía de resonancia, la desviación isomérica y los estados de oxidación (Fleischer, 2010). En el caso de los estados de oxidación no definidos presentan una gran anchura espectral (Stevens et al., 2005).

\section{Discusión}

En las distintas zonas de estudio se han detectado especies minerales de carácter primario y secundario, así como también materiales accesorios. Los olivinos detectados son mayoritariamente forsterita en todas las zonas, lo cual confirma lo señalado por otros autores en la bibliografía especializada y por las diferentes técnicas empleadas. Los piroxenos se corresponden a fases de augita y diópsido, donde la discriminación espectral y desplazamientos en los espectros Raman es mínima (Wang et al. 2001), confirmándose que no hay gran variación de cationes $\left(\mathrm{Ca}^{2+}, \mathrm{Mg}^{2+}, \mathrm{Fe}^{2+}\right)$ presentes en la estequiometría.
Los feldespatos detectados podrían ser de carácter primario, aunque algunos como es el caso de la albita, podrían haber sido generados por procesos de alteración hidrotermal a temperaturas inferiores a $300^{\circ} \mathrm{C}$ (Bustillo \& Martínez-Frías, 2003; RodríguezLosada et al., 2000).

Los óxidos en las tres zonas estudiadas presentan una gran cantidad de similitudes desde el punto de vista espectral. Pero, mediante espectroscopia Mössbauer se han podido establecer diferencias que podrían esclarecer su origen (Klingelhofer et al., 1992).

En la Tabla 6 se presenta una recopilación de la mineralogía marciana en comparación con las especies minerales encontrada en los afloramientos de Tenerife estudiados (Chevrier, 2007; Schmidt et al., 2009, Bish et al., 2013). Los minerales detallados en la Tabla 6 han sido identificados tanto por los Rovers como a través de estudios realizados sobre meteoritos marcianos como los SNC (Shergotitas-NakhalitasChassignitas) (Bridges et al., 2001; Mittlefehldt, 1999). En el caso de los materiales marcianos de alteración se barajan tres hipótesis sobre su origen: alteración hídrica y procesos evaporíticos; hidrotermalismo; y procesos de meteorización (Chevrier, 2007). Los depósitos de materiales sedimentarios y alterados en el Meridianni Planum de Marte podrían compararse con la zona de Las Cañadas o el Malpaís (Carr, 2007; Lalla, 2014). Esta caldera representa sin duda una zona de interés como análogo, tanto desde el punto de vista de su geomorfología como en cuanto a su petrología, geoquímica y procesos de alteración (Horgan, 2013).

La técnica Raman, tanto en el ámbito microscópico como su futura aplicación in situ-espacial, ha permitido identificar la mayor parte de las especies minerales primarias, secundarias y minerales accesorios (Rull \& Martínez-Frías, 2006; Rull et al., 2011). Los análisis realizados sobre las muestras, mediante DRX y Mössbauer, fueron comparados con los obtenidos en Marte a través de la instrumentación espacial gemela funcionando en los Rovers marcianos, por ejemplo en caso de DRX mediante el sistema CheMin en MSL (Mar Science Laboratory) y en el caso de espectroscopia Mössbauer mediante los resultados obtenidos en el sistema MIMOS-II del Laboratorio Athenas de las misiones MER (Mars Exploration Rover) (Fleischer, 2010). Los resultados apuntan a una gran similitud con la mineralogía marciana (Bish, et al., 2013). 
Tabla 6.-Comparativa mineralógica entre Marte y las zonas estudiadas (La Caldera de las Cañadas, erupciones históricas y afloramiento del Malpaís) considerando las especies más abundantes

\begin{tabular}{ll}
\hline Minerales hallados en Marte & Minerales estudiados en los afloramientos \\
\hline Olivinos & Olivinos (Forsterita y Monticellita) \\
Piroxenos (con alto contenido en Ca), Clino-piroxenos y Orto-piroxenos & Piroxenos (Augita y Diópsido) \\
Plagioclasas & Plagioclasas y Feldespatos alcalinos \\
Filosilicatos (Caolinita, Serpentina, Nontronita, Monmorillonita, etc) & Filosilicatos (Caolinita, Moscovita e Illita) \\
Óxidos de Hierro (Hematite, Goethita y Magnetita) & Óxidos de Hierro (Hematite, Goethita y Magnetita) \\
Sulfatos de Hierro & \\
Carbonatos (Carbonatos Ca-Fe-Mg y Siderita) & Carbonatos (Calcita) \\
\hline
\end{tabular}

\section{Conclusión}

Se ha realizado un estudio de muestras de los tres afloramientos mediante dos técnicas aplicadas en la exploración espacial (difracción de rayos X y espectroscopia Mössbauer) en los instrumentos gemelos usados en las misiones MER y MSL. Además, se recurrió a la espectroscopia Raman, que ha demostrado ser una herramienta muy potente para la identificación mineralógica y para el estudio de los distintos procesos de alteración dependiendo del entorno geológico. Por otro lado, la espectroscopia Raman presenta otras ventajas al ser una técnica no-destructiva a escala del nano-grano mineral y destacándose su capacidad de detección de compuestos orgánicos (material carbonáceo). De esta manera, se puede indicar que el uso de técnicas espectroscópicas de carácter vibracional, en especial la técnica Raman, será de fundamental importancia en el cumplimiento de los objetivos de la misión ESA-ExoMars.

Los resultados mineralógicos muestran la presencia de olivinos, piroxenos, plagioclasas y materiales de carácter secundario, que también han sido detectados en Marte, meteoritos marcianos y otros análogos. La zona de las Cañadas posee una variedad mineralógica mayor debido a que es una de las zonas más antiguas, habiendo sufrido mayor cantidad de procesos de alteración sobre sus afloramientos. Por otro lado, las erupciones históricas podrían servir de análogo marciano para el volcanismo más primitivo de Marte, ya que no presenta apenas alteraciones. Por otro, las fases de oxidación, mediante espectroscopia Mössbauer, se han podido diferenciar pudiéndose establecer su estado de oxidación.

Dada la gran cantidad de muestras analizadas, variedad de especies y variedad de procesos en los afloramientos es necesario realizar estudios pormenorizados sobre cada muestra mediante un mapeo Raman. De esta manera, se puede obtener información punto a punto del grano-mineral y de su entorno mineral más próximo brindando pistas e indicios de los orígenes alteracionales de los materiales secundarios. En futuros análisis mediante mapeos con sistemas Raman se podrá establecer el tipo de alteración (serpentinización, etc.).

\section{AGRADECIMIENTOS}

Los autores agradecen al MINECO por el apoyo económico prestado mediante los proyectos de investigación AYA200804529-ES y AYA2011-30291-C02-01. El primer autor desea agradecer al Ministerio de Innovación la beca FPI-2009 y la ayuda económica recibida para el desarrollo de la estancia de investigación en la Universidad de Mainz. Los autores agradecen al Dr. José Antonio Rodríguez Losada de la Universidad de la Laguna por su apoyo y organización en las distintas campañas realizadas, como así también a Francisco GarcíaTalavera Casañas del Museo de Ciencias Naturales de Tenerife. Además, también desean agradecer a los revisores anónimos por las revisiones y sugerencias hechas correspondientes al manuscrito.

\section{Referencias}

Agee, C.B.; Wilson, N.V.; McCubbin, F.M.; Ziegler, K.; Polyak, V.J.; Sharp, Z.D.; Asmerom, Y.; Nunn, M.H.; Shaheen, R.; Thiemens, M.H.; Steele, A.; Fogel, M.L.; Bowden, R.; Glamoclija, M.; Zhang, Z. \& Elardo, S.M. (2013). Unique Meteorite from Early Amazonian Mars: Water-Rich Basaltic Breccia Northwest Africa 7034. Science, 339 (6121): 780-785. http://dx.doi.org/ $10.1126 /$ science. 1228858

Anguita, F.A. \& Hernán, F. (1975). A propagating fracture model versus a hot spot origin for the Canary Islands. Earth and Planetary Science Letters, 27 (1): 11-19. http://dx.doi.org/10.1016/0012-821X(75)90155-7 
Anguita, F. \& Hernán, F. (2000). The Canary Islands origin; a unifying model. Journal of Volcanology and Geothermal Research, 103: 1-26. http://dx.doi.org/ 10.1016/S0377-0273(00)00195-5

Apopei, A.I. \& Buzgar, N. (2010). The Raman study of amphiboles. Analele ştiințifice ale Universităţii “Al. i. cuza" Iaşi, Geologie, 56 (1): 57-83.

Araña, V. (1979). Litología y estructura del Edificio Cañadas, Tenerife (Islas Canarias). Estudios geológicos, 27: $95-135$.

Araña, V. \& Ortiz, A. (1986). La volcanología Actual: Una revisión. Anales de Física, 82: 1-14.

Barnes, D.; Battistelli, E.; Bertrand, R.; Butera, F.; Chatila, R.; Del Biancio, A.; Draper, C.; Ellery, A.; Gelmi, R.; Ingrand, F.; Koeck, C.; Lacroix, S.; Lamon, P.; Lee, C.; Magnani, P.; Patel, N.; Pompei, C.; Re, E.; Richter, L.; Rowe, M.; Siegwart, R.; Slade, R.; Smith, M.F.; Terrien, G.; Wall, R.; Ward, R.; Waugh, L. \& Woods, M. (2006). The ExoMars rover and Pasteur payload Phase A study: an approach to experimental astrobiology. International Journal of Astrobiology, 5 (3): 221-241. http://dx.doi.org/10.1017/S1473550406003090

Bish, D.L.; Carey, J.W.; Vaniman, D.T. \& Chipera, S.J. (2003). Stability of hydrous minerals on the Martian surface. Icarus, 164: 96-103. http://dx.doi.org/10.1016/ S0019-1035(03)00140-4

Bish, D.L.; Blake, D.; Sarrazin, P.; Treiman, A.H.; Hoehler, T.; Hausrath, E.M., Midtkandal, I. \& Steele, A. (2007). Field XRD/XRF mineral analysis by the MSL CheMin instrument. 38th Lunar and Planetary Science Conference, Abstract, 1163.

Bish, D.L.; Blake, D.F.; Vaniman, D.T.; Chipera, S.J.; Morris, R.V.; Ming, D.W.; Treiman, A.H.; Sarrazin, P.; Morrison, S.M.; Downs, R.T.; Achilles, C.N.; Yen, A.S. Bristow, T.F.; Crisp, J.A.; Morookian, J.M.; Farmer, J.D.; Rampe, E.B.; Stolper, E.M.; Spanovich, N. \& MSL Science Team (2013). X-ray Diffraction Results from Mars Science Laboratory: Mineralogy of Rocknest at Gale Crater. Science, 341 (6153): 1238932. http://dx.doi.org/10.1126/science. 1238932

Bridges, J.C.; Catling, D.C.; Saxton, J.M.; Swindle, T.D.; Lyon, I.C. \& Grady, M.M. (2001). Alteration assemblages in Martian meteorites: implications for near-surface processes. Space Science Review, 96: 365-392. http://dx.doi.org/10.1023/A: 1011965826553

Bustillo, M.A. \& Martínez-Frías, J. (2003). Green Opals in Hydrothermalized basalts (Tenerife Island, Spain): Alteration and aging of silica pseudoglass. Journal of non-crystalline solids, 323: 27-33. http://dx.doi. org/10.1016/S0022-3093(03)00288-6

Carr, M.H. \& Greeley, R. (1980). Volcanic features of Hawaii: A basis for comparison with Mars. NASA SP, $403.211 \mathrm{p}$.

Carr, M.H. (2007). The Surface of Mars. Cambridge University Press, Cambridge, 322 p.
Carracedo, J. (1975). Estudio paleomagnetico de la isla de Tenerife (islas Canarias). Tesis Doctoral, Universidad Complutense, $265 \mathrm{p}$.

Carracedo, J. (1999). Growth, structure, instability and collapse of Canarian volcanoes and comparisons with Hawaiian volcanoes. Journal of Volcanology and Geothermal Research, 94 (1-4): 1-19. http://dx. doi.org/10.1016/S0377-0273(99)00095-5

Chevrier, V. (2007). Mineralogy and evolution of the Surface of Mars: A review. Planetary and Space Science, 55 (3): 289-314. http://dx.doi.org/10.1016/j. pss.2006.05.039

Chopelas, A. (1999). Estimates of mantle relevant Clapeyron slopes in the $\mathrm{MgSiO}_{3}$ system from high-pressure spectroscopic data. American Mineralogist, 84 (3): 233-244.

Christensen, P.R.; Bandfield, J.L.; Bell III, J.F.; Gorelick, N.; Hamilton, V.E.; Ivanov, A.; Jakosky, B.M.; Kieffer, H.H.; Lane, M.D.; Malin, M.C.; Mehall, G.L.; McConnochie, T.; McEwen, A.S.; McSween, H.Y.; Moersch, J.E.; Nealson, K.H.; Rice, J.W.; Richardson, M.I.; Ruff, S.W.; Smith, M.D.; Titus, T.N. \& Wyatt, W. (2003). Morphology and composition of the surface of Mars: Mars Odyssey THEMIS results. Science, 300 (5628): 2056-2061. http://dx.doi.org/10.1126/ science. 1080885

Dong Zu, E.; Qing Li, S.; Zou, Y.; Gang Zhao, X.; Dan Sun, Y.; Fei Lin, Y. \& Li, H. (2011). Study of Natural and Synthetic Quartz by Raman Spectra. Key Engineering Materials, 492: 341-344. http://dx.doi.org/10.4028/ www.scientific.net/KEM.492.341

Donoghue, E. \& Troll, V.R. (2008). Low temperature hydrothermal alteration of intra-caldera tuffs, Miocene Tejeda. Journal of Volcanology and Geothermal Reseach, 176(4): 551-564.http://dx.doi.org/10.1016/j. jvolgeores.2008.05.002

Ellery, A.; Kolb, C.; Lammer, H.; Parnell, J.; Edwards, H.; Richter, L.; Patel, M.; Romstedt, J.; Dickensheets, D.; Steele, A. \& Cockell, C. (2003). Astrobiological instrumentation for Mars - the only way is down. International Journal of Astrobiology, 1 (4): 365-380. http://dx.doi.org/10.1017/S1473550403001204

Fleischer, I. (2010). Diversity of Lithologic Components at Meridiani Planum, Mars: Insights from Mössbauer Spectroscopic Investigations. Tesis Doctoral Johannes Gutenberg-Universität an Mainz.

Freeman, J.J.; Wang, A.; Kuebler, K.E.; Jolliff, B.L. \& Haskin, L.A. (2008). Characterization of natural feldspars by Raman spectroscopy for future planetary exploration. The Canadian Mineralogist, 46 (6): 1477-1500. http://dx.doi.org/10.3749/canmin.46.6.1477

Frost, R.L. \& Kloprogge, J.T. (2001). Towards a single crystal Raman spectrum of kaolinite at $77 \mathrm{~K}$. Spectrochimica Acta Part A, 57 (1): 163-175. http://dx. doi.org/10.1016/S1386-1425(00)00345-0

García-Hernández, J.E.; Notario Del Pino, J.S.; González Martín, M.M.; Hernán Reguera, F. \& Rodríguez 
Losada, J.A. (1993). Zeolites in pyroclastic deposits in southeastern Tenerife (Canary Islands). Clays and Clay Minerals, 41 (5): 521-526.

Haley, L.V.; Wylie, I.W. \& Koningstein, J.A. (1982). An investigation of the lattice and interlayer water vibrational spectral regions of muscovite and vermiculite using Raman microscopy. Journal of Raman Spectroscopy, 13 (2): 203-205. http://dx.doi.org/10.1002/ jrs. 1250130217

Hofmeister, A.M.; Cynn, H.; Burnley, P.C. \& Meade, C. (1999). Vibrational spectra of dense, hydrous magnesium silicates at high pressure: Importance of the hydrogen bond angle. American Mineralogist, 84: 454-464.

Horgan, B. (2013). Planetary science: Evolved magma on Mars. Nature Geoscience, 6: 991-992. http://dx.doi. org/10.1038/ngeo2010

Houghton, B.; Rymer, H.; Stix, J. \& McNutt, S. (1999). Encyclopedia of Volcanoes. Academic Press, $1417 \mathrm{p}$.

Huang, E; Chen, C.H.; Huang, T.; Lin, E.H. \& Xu, JI-AN. (2000). Raman spectroscopic characteristics of $\mathrm{Mg}-\mathrm{Fe}-\mathrm{Ca}$ pyroxenes. American Mineralogist, 85 (3-4): 473-479.

Jubb, A.M. \& Allen, H.C. (2010). Vibrational Spectroscopic characterization of the Hematite, Maghemite and magnetite thin films produced by Vapor deposition. Applied Materials \& Interfaces, 2 (10): 2804-2812. http://dx.doi.org/10.1021/am1004943

Klingelhöfer, G.; Imkeller, U.; Kankeleit, E. \& Stahl, B. (1992). Remarks on depth selective CEMS Backscattering measurements. Hyperfine Interactions, 71: 1445-1448. http://dx.doi.org/10.1007/BF02397355

Klingelhöfer, G.; Morris, R.V.; Bernhardt, B.; Rodionov, D.; de Souza, P.A.; Squyres, S.W.; Foh, J.; Kankeleit, E.; Bonnes, U.; Gellert, R.; Schröder, C.; Linkin, S.; Evlanov, E.; Zubkov, B. \& Prilutski, O. (2003). Athena - MIMOS II Mössbauer spectrometer investigation. Journal of Geophysical Research: Planets, 108 (E12): 8067. http://dx.doi.org/10.1029/2003JE002138

Klingelhöfer, G.; Blumers, M.; Bernhardt, B.; Lechner, P.; Gironés-Lopez, J.; Maul, J.; Soltau, H.; Strüder, L. \& Henkel, H. (2010). The improved miniaturised Mössbauer spectrometer MIMOS IIA with elemental analysis capability and increased sensitivity. 41st Lunar and Planetary Science Conference, Abstract, 2423.

Kloprogge, J.T.; Hickey, L. \& Frost, R.L. (2002). Synthesis and spectroscopic characterisation of deuterated hydrotalcite. Journal of Materials Science Letters, 21 (8): 603-605. http://dx.doi.org/10.1023/A: 1015655018529

Krisshnamurti, D. (1962). The Raman Spectrum of rutile. Proceedings of the Indian Academy of Sciences, 55 (5): 290-299. http://dx.doi.org/10.1007/BF03045870

Kuebler, K.E.; Jolliff, B.L.; Wang, A. \& Haskin, L.A. (2006). Extracting olivine ( $\mathrm{Fo}-\mathrm{Fa}$ ) compositions from Raman spectral peak positions. Geochimica et Cosmochimica Acta, 70 (24): 6201-6222. http:// dx.doi.org/10.1016/j.gca.2006.07.035

Lalla, E.; Sansano, A.; Sanz, A.; Alonso, P.; Medina, J.; Martínez-Frías, J. \& Rull, F. (2010). Espectroscopia Raman de Basaltos Correspondientes al Volcán de las Arenas, Tenerife. Macla, 13: 129-130.

Lalla, E.; Sansano, A.; Sanz, A.; Navarro, R.; LópezReyes, G.; Venegas, G.; Rodríguez, J.A.; Medina, J.; Martínez-Frías, J; Rull, F. (2011). Raman spectroscopy of Pillow Lavas from the Anaga Zone - Tenerife, Canary Island. Macla, 15: 119-120.

Lalla, E. (2014). Tenerife como análogo de Marte: Caracterización multi-analítica (Raman, DRX, ATR-FTIR, SEM y Mössbauer) de muestras de interés planetario y Astrobiológico. Tesis Doctoral, Universidad de Valladolid. 214 p. http://uvadoc.uva.es/handle/ $10324 / 4572$

Martí, J.; Hurlimann, M.A. \& Gudmundsson, G.J. (1997). Stratigraphy, structure and geochronology of the Las Cañadas caldera (Tenerife, Canary Islands). Geological Magazine, 131 (6): 715-727. http://dx.doi.org/ $10.1017 / \mathrm{S} 0016756800012838$

Martí, J. \& Gudmundsson, G.J. (2000). The Cañadas caldera (Tenerife, Canary Islands): An ovverlapping collapse caldera generated by a magma-chamber migration. Journal of vulcanology and geothermal research, 103: 161-173. http://dx.doi.org/10.1016/ S0377-0273(00)00221-3

Mckeown, D.A.; Bell, M.I. \& Etz, E.S. (1999). Vibrational Analysis of the Dioctahedral Mica: 2M1 Muscovite. American Mineralogist, 84: 1041-1048.

McSween, H.Y.; Taylor, G.J. \& Wyatt, M.B. (2009). Elemental composition of the Martian crust. Science, 324 (5928): 736-739. http://dx.doi.org/10.1126/science. 1165871

Mittlefehldt, D.W. (1994). ALH84001, a cumulate orthopyroxenite member of the Martian meteorite. Meteoritics, 29 (2): 214-221. http://dx.doi.org/ 10.1111/j.1945-5100.1994.tb00673.x

Mouginis-Mark, P. \& Robinson, M.S. (1992). Evolution of the Olympus Mons Caldera, Mars. Bulletin of Volcanology, 54 (5): 347-360. http://dx.doi.org/10.1007/ BF00312318

Mouri, T. \& Enami, M. (2008). Raman spectroscopic study of olivine-group minerals. Journal of Mineralogical and Petrological Science, 103: 100-104. http://dx.doi. org/10.2465/jmps.071015

Mustard, J.F.; Murchie, S.L.; Pelkey, S.M.; Ehlmann, B.L.; Milliken, R.E.; Grant, J.A.; Bibring, J.P.; Poulet, F.; Bishop, J.; Noe Dobrea, E.; Roach, L.; Seelos, F.; Arvidson, R.E.; Wiseman, S.; Green, R.; Hash, C.; Humm, D.; Malaret, E.; McGovern, J.A.; Seelos, K.; Clancy, T.; Clark, R.; Marais, D.D.; Izenberg, N.; Knudson, A.; Langevin, Y.; Martin, T.; McGuire, P.; Morris, R.; Robinson, M.; Roush, T.; Smith, M.; Swayze, G.; Taylor, H.; Titus, T. \& Wolff, M. (2008). Hydrated silicate minerals on Mars observed by the 
Mars Reconnaissance Orbiter CRISM instrument. Nature, 454: 305-309. http://dx.doi.org/10.1038/ nature 07097

Okada, T.; Narita, T.; Nagai, T. \& Yamanaka, T. (2008). Comparative Raman spectroscopic study on ilmenitetype $\mathrm{MgSiO}_{3}$ (akimotoite), $\mathrm{MgGeO}_{3}$, and $\mathrm{MgTiO}_{3}$ (geikielite) at high temperatures and high pressures. American Mineralogist, 93 (1): 39-47. http://dx.doi. org/10.2138/am.2008.2490

Osinski, G.R.; Léveillé, R.; Berinstain, A.; Lebeuf, M. \& Bamsey, M. (2006). Terrestrial Analogues to Mars and the Moon: Canada's Role. Geoscience Canada, 33 (4): $175-188$.

Risueño-Díaz, E. (2005). Reserva Natural especial del Malpaís de Guímar: Informe del Plan director. Consejería de medio ambiente y Ordenación Territorial, Gobierno de Canarias, 55 p.

Rodríguez-Losada, J.A.; Martínez-Frías, J.; Bustillo, M.A.; Delgado, A.; Hernández-Pacheco, A. \& De la Fuente Krauss, J.V. (2000). The hydrothermally altered ankaramite basalts of Punta Poyata (Tenerife, Canary Islands). Journal of Volcanology and Geothermal Research, 103: 367-376. http://dx.doi.org/10.1016/ S0377-0273(00)00231-6

Rodríguez-Badiola, E. \& Carracedo, J.C. (2008). El volcán Teide. Tomo I, Geología y volcanología del Teide y las dorsales. Rocas Volcánicas del Teide. Excmo. Cabildo de Santa Cruz de Tenerife, Consejería de Medio Ambiente, 145 p.

Romero, C; Quirantes, F. \& Martínez de Pisón, E. (1986). Los Volcanes, guía física de España 1. Alianza Editorial. 254 p.

Rull, F. \& Martínez-Frías, J. (2003). Identification of Calcite grains in the Vaca Muerta mesosiderite by Raman Spectroscopy. Journal of Raman Spectroscopy, 34 (5): 367-370. http://dx.doi.org/10.1002/jrs. 1003

Rull, F. \& Martínez-Frías, J. (2006). Raman spectroscopy goes to mars. Spectroscopy Europe, 18 (1): 18-21. http://hdl.handle.net/10261/36075

Rull, F.; Martínez-Frías, J. \& Rodríguez-Losada, J.A. (2007). Micro-Raman spectroscopic study of El Gasco pumice, western Spain. Journal of Raman Spectroscopy, 38 (2): 239-244. http://dx.doi.org/10.1002/ jrs. 1628

Rull, F.; Maurice, S.; Díaz, E.; Tato, C.; Pacros, A. \& RLS Team (2011). The Raman Laser Spectrometer (RLS) on the ExoMars 2018 Rover Mission. 42nd Lunar and Planetary Science Conference, Abstract, 1608.

Schmidt, M.E.; Farrand, W.H.; Johnson, J.R.; Schröder, C.; Hurowitz, J.A.; McCoy, T.J.; Ruff, S.W.; Arvidson, R.E.; Des Marais, D.J.; Lewis, K.W.; Ming, D.W.; Squyres, S.W. \& de Souza, P.A. (2009). Spectral, mineralogical, and geochemical variations across Home Plate, Gusev Crater, and Mars indicate high and low temperature alteration. Earth and Planetary Science Letters, 281 (3-4): 258-266. http://dx.doi. org/10.1016/j.epsl.2009.02.030
Sekiya, T.; Ohta, S.; Kamei, S.; Hanakawa, M. \& Kurita, S. (2001). Raman spectroscopy and phase transition of anatase $\mathrm{TiO}_{2}$ under high pressure. Journal of Physics and Chemistry Solids, 62 (4): 717-721. http:// dx.doi.org/10.1016/S0022-3697(00)00229-8

Sharma, S.K.; Lucey, P.G.; Ghosh, M.; Hubble, H.W. \& Horton, K.A. (2003) Stand-off Raman spectroscopic detection of minerals on planetary surfaces. Spectrochimica Acta Part A: Molecular and Biomolecular Spectroscopy, 59 (10): 2391-2407. http://dx.doi. org/10.1016/S1386-1425(03)00080-5

Stalport, F.; Glavin, D.P.; Eigenbrode, J.L.; Bish, D.; Blake, D.; Coll, P.; Szopa, C.; Buch, A.; McAdam, A.; Dworkin, J.P. \& Mahaffy, P.R. (2012). The influence on mineralogy on recovering organic acids from Mars analogue materials using the "one-pot" derivatization experiment on the Sample Analysis at Mars (SAM) instrument suite. Planetary and Space Science, 67 (1): 1-13. http://dx.doi.org/10.1016/j.pss.2012.02.010

Stevens, J.G; Khasanov, A.M.; Miller-Bill, J.W.; Pollak, H. \& Zhe, Li. (2005). Mössbauer Mineral Handbook. University of North Carolina at Asheville, North Carolina, $636 \mathrm{p}$.

Stolper, E.M.; Baker, M.B.; Newcombe, M.E.; Schmidt, M.E.; Treiman, A.H.; Cousin, A.; Dyar, M.D.; Fisk, M.R.; Gellert, R.; King, P.L.; Leshin, L.; Maurice, S.; McLennan, S.M.; Minitti, M.E.; Perrett, G.; Rowland, S.; Sautter, V.; Wiens, R.C. \& MSL Science Team (2013). The Petrochemistry of Jake M: A Martian Mugearite. Science 27, 341 (6153): 1239463. http:// dx.doi.org/10.1126/science. 1239463

Thirlwall, M.F.; Singer, B.S. \& Marriner, G.F. (2000). $39 \mathrm{Ar}-40 \mathrm{Ar}$ ages and geochemistry of the basaltic shield stage of Tenerife, Canary Islands, Spain. Journal of Volcanology and Geothermal Research, 103 (1-4): 247-297. http://dx.doi.org/10.1016/S03770273(00)00227-4

Vago, J. (2006). ExoMars: Searching for life on the Red Planet. ESA Bulletin, 126: 16-23.

Wang, A., Haskin, L.A., Lane, A.L., Wdowiak, T.J., Squyres, S.W., Wilson, R.J., Hovland, L.E., Manatt, K.S., Raouf, N. \& Smith, C.D. (2003). Development of the Mars microbeam Raman spectrometer (MMRS). Journal of Geophysical. Research: Planets, 108 (E1): 5005. http://dx.doi.org/10.1029/2002JE001902

West, M.D.; Clarke, J.D.A.; Thomas, M.; Pain, C.F. \& Walter, M.R. (2010). The geology of Australian Mars analogue sites. Planetary and Space Science, 58 (4): 447-458. http://dx.doi.org/10.1016/j.pss.2009.06.012

Wang, A.; Jolliff, B.L.; Haskin, L.A.; Kuebler, K.E. \& Viskupic, K.M. (2001). Characterization and comparison of structural and compositional features of planetary quadrilateral pyroxenes by Raman spectroscopy. American Mineralogist, 86 (7-8): 790-806.

Wray, J.J.; Hansen, S.T.; Dufek, J.; Swayze, G.A.; Murchie, S.L.; Seelos, F.P.; Skok, J.R.; Irwin III, R.P. \& Ghiorso, M.Z. (2013). Prolonged magmatic 
activity on Mars inferred from the detection of felsic rocks. Nature Geoscience 6, 1013-1017. http://dx. doi.org/10.1038/ngeo1994

Zattina, M.; Bersani, D. \& Carter, A. (2007). Raman microspectroscopy: Anon-destructive tool for routine calibration of apatite crystallographic structure for fission-track analyses. Chemical Geology, 240 (3-4): 197-204. http://dx.doi.org/10.1016/j.chemgeo.2007. 02.007
Zoltai, T. \& Scout, J.H. (1984). Mineralogy: Concepts and Principles. Burgess Publishing Co., Mineapolis. $505 \mathrm{p}$.

Zotov, N. \& Ebbsjo, I. (1999). Calculation of Raman spectra and vibrational properties of silicate glasses: Comparison between $\mathrm{Na}_{2} \mathrm{Si}_{4} \mathrm{O}_{9}$ and $\mathrm{SiO}_{2}$ glasses. Physical Review B, 60 (9): 6383-6397. http://dx.doi. org/10.1103/PhysRevB.60.6383 